\title{
Toward de Sitter space from ten dimensions
}

\author{
Jakob Moritz, ${ }^{*}$ Ander Retolaza, ${ }^{\dagger}$ and Alexander Westphal ${ }^{\dagger}$ \\ Deutsches Elektronen-Synchrotron, DESY, Notkestraße 85, 22607 Hamburg, Germany
}

(Received 4 December 2017; published 22 February 2018)

\begin{abstract}
Using a 10D lift of nonperturbative volume stabilization in type IIB string theory, we study the limitations for obtaining de Sitter vacua. Based on this we find that the simplest Kachru, Kallosh, Linde, and Trivedi vacua with a single Kähler modulus stabilized by a gaugino condensate cannot be uplifted to de Sitter. Rather, the uplift flattens out due to stronger backreaction on the volume modulus than has previously been anticipated, resulting in vacua which are metastable and supersymmetry breaking, but that are always anti-de Sitter (AdS). However, we also show that setups such as racetrack stabilization can avoid this issue. In these models it is possible to obtain supersymmetric AdS vacua with a cosmological constant that can be tuned to zero while retaining finite moduli stabilization. In this regime, it seems that de Sitter uplifts are possible with negligible backreaction on the internal volume. We exhibit this behavior also from the $10 \mathrm{D}$ perspective.
\end{abstract}

DOI: 10.1103/PhysRevD.97.046010

\section{INTRODUCTION}

The cosmological constant (c.c.) problem stands as our perhaps most drastic disagreement between theory and observation. When applying quantum field theory (QFT) to various stages of symmetry breaking and to the Einstein field theory limit of gravity given by general relativity (GR), we arrive at a prediction which is off by some 120 orders of magnitude from the value of the c.c. inferred from, for example, precision observations of the cosmic microwave background (CMB).

Attempting to resolve this tension is difficult given a nogo theorem by Weinberg [1] which essentially states that dynamic adjustment mechanisms based on a fully local field theory only must fail. This leaves us with few options.

One possibility is to give up locality, motivated by the fact that a true c.c. needs the full 4-volume of the Universe for a proper detection, which makes it borderline nonlocal. Recent work has shown that a combination of protective scale and shift symmetries with the introduction of certain effectively global variables encoded via four-form field strengths can "sequester" the QFT vacuum energy from its gravitating effects [2-4].

\footnotetext{
*jakob.moritz@desy.de

ander.retolaza@desy.de

*alexander.westphal@desy.de
}

Published by the American Physical Society under the terms of the Creative Commons Attribution 4.0 International license. Further distribution of this work must maintain attribution to the author(s) and the published article's title, journal citation, and DOI. Funded by SCOAP.
Another possibility, already foreseen by Linde, Weinberg, and others [5-9], is to replace the QFT limit of GR with a full candidate theory of quantum gravity, and assume that it will render the quantum vacuum energy finite and generically large, but produce an enormous number of different solutions (vacua) with differing finite c.c.'s. If we couple this with a cosmological population mechanism such as eternal inflation [10,11], then a form of a weak anthropic argument can explain the observed c.c. value as natural given certain observed features such as structure formation, and provided the number of different vacua is larger than $\mathcal{O}\left(\Lambda^{-4}\right)=\mathcal{O}\left(10^{120}\right)$ (and the distribution of vacuum energies shows no strong features).

Crucial for this line of argument is having in hand such a candidate quantum gravity with an enormous "landscape" of discrete vacua with varying c.c. values [12].

The first serious and still one of the best candidates for this task is the setup by Kachru, Kallosh, Linde, and Trivedi (KKLT) [13] which uses string theory as a candidate quantum gravity to realize a version of the BoussoPolchinski proposal, a toy model of the landscape solution to the c.c. problem.

KKLT propose to generate de Sitter vacua in string theory in a three-step process:

(i) Step 1: Stabilize complex structure moduli and the axio-dilaton perturbatively using three-form fluxes $[14,15]$.

(ii) Step 2: Stabilize Kähler moduli using nonperturbative effects. The result is a supersymmetric AdS vacuum [13].

(iii) Step 3: Break supersymmetry and lift to de Sitter space by including an antibrane at the bottom of a warped throat $[13,16]$. 
In looking at these three steps we observe the following situation. The 4D effective supergravity description of step 1 is derived via dimensional reduction from the warped Calabi-Yau (CY) compactifications with imaginary selfdual (ISD) three-form fluxes, and its critical points match with solutions of the full 10D equations of motion, the 10D Einstein equations, and Bianchi identities supplemented by $O 3 / O 7$ planes and $D 3 / D 7$-branes as local sources. The volume modulus $\rho$ is a flat direction at this level [14].

The 4D effective description of step 2 is based on the 4D effective theory of gaugino condensation [17-20]. However, the presence of unbroken supersymmetry in the ensuing AdS vacuum of $\rho$ as well as the suppression of the $\rho$ mass scale with the control parameter $\left|W_{0}\right|$ given by the fluxes provides justification for the choice of the form of the $4 \mathrm{D}$ effective field theory (EFT) of the $\rho$ modulus, as it is more or less dictated by holomorphy of the superpotential, supersymmetry of the setup, nonperturbative gauge dynamics, and the relation between the holomorphic gauge kinetic function of the $D 7$-brane gauge theory and the four-cycle volume modulus chiral multiplet $f_{D 7}\left(\Sigma_{4}^{i}\right) \sim \rho_{i}$. Moreover, all scales of the volume stabilization process lie far below those of the flux stabilization of step 1 [13].

There is by now very strong evidence that step 3, inserting in particular a single anti- $D 3$-brane at the bottom of the warped throat of step $1+$ step 2, is still a wellcontrolled metastable solution of the full system with spontaneously broken supersymmetry. Its nonperturbative instability arises from the Kachru-Pearson-Verlinde (KPV) flux-brane annihilation process [16,21]. Describing this system beyond the probe approximation for the anti-D3brane [22-33] while respecting a well-defined EFT description of the anti-D3 backreaction onto the warped throat geometry, with correct matching onto the string scale physics $[34,35]$ provides clear arguments that the KPV process is the microscopic nonperturbative instability of the setup with all perturbative directions (the anti-D3 position moduli) frozen.

However, we observe a more subtle peculiarity of this uplift effect in the KKLT setting. Namely, the amount of uplift necessary to reach de Sitter is tied to the mass scale of the $\rho$ modulus from its stabilization in step 2. To reach de Sitter we require validity of a particular form of the 4D EFT. Modifications to the EFT that are compatible with our current knowledge of the supersymmetric (SUSY) moduli stabilization as well as the classical $\overline{D 3}$-brane potential can lead to substantial backreaction effects on the volume modulus. In the extreme case these modifications could lead to a flattened uplift that never reaches a positive vacuum potential. "Flattening" means that the backreacted uplift turns out to be smaller than the estimate without backreaction which scales linear with the antibrane tension.

We note here that such flattening effects from moduli backreaction were observed before in a related context: namely, if we replace the control parameter of the uplifting vacuum energy, the antibrane tension, by the potential energy of a slow-rolling scalar field driving inflation, the same backreaction effects lead to flattened scalar potentials for inflation [36], in complete analogy with the uplift flattening we observe here.

Indeed such modifications to the EFT can be argued to arise through the same effects that lead to the lifting of the moduli space of $D 3$-brane position moduli by nonperturbative effects. However, the very same reasoning suggests that if the racetrack stabilization of Kallosh and Linde [37] is assumed, de Sitter uplifts can be realized because the AdS depth of the supersymmetric potential can be parametrically decoupled from the mass scale of the Kähler modulus.

Consequently, the questions we would like to ask and partially answer in this paper in the course of Secs. II through IV are as follows. Is the volume stabilization of the original KKLT proposal rigid enough to allow for a de Sitter uplift or is racetrack stabilization a minimal requirement? If the latter is true, can we verify the stabilization mechanism also from a 10D perspective?

We find indeed that backreaction effects play a substantially stronger role than has previously been anticipated. The uplift flattening we observe is strong enough that uplifts reaching de Sitter are not possible using the simplest version of KKLT. Instead the solutions for volume stabilization with a single gaugino condensate are metastable SUSY-breaking AdS vacua. However, assuming certain properties of the compactification manifold, we are able to give a 10D picture of racetrack stabilization that strongly indicates the possibility to uplift to de Sitter in this case.

We use these 4D insights to build a modified singlecondensate moduli stabilization scheme by including $\alpha^{\prime}$ corrections. This allows us to decouple the mass scale of the Kähler modulus from the AdS depth, although not parametrically.

We then discuss our findings and conclude in Sec. VI.

\section{MODULI STABILIZATION AND DE SITTER UPLIFTS IN TYPE IIB STRING THEORY: THE 4D PERSPECTIVE}

The best understood constructions of de Sitter vacua in type IIB string theory start from Calabi-Yau (CY) compactifications to 4D Minkowski and orientifolds thereof. These compactifications come with many massless moduli which need to be stabilized in order to make them phenomenologically interesting and typically require some extra structure/ingredient to give rise to 4D de Sitter. This program of "moduli stabilization" and de Sitter uplift usually is carried out in three steps:

(i) Stabilization of complex structure moduli and the axiodilaton at tree level by introduction of threeform fluxes [14]. 
(ii) Stabilization of Kähler moduli. Different approaches exist to stabilize them, probably the most common ones being the following: in the so-called KKLT approach [13], one stabilizes them using nonperturbative effects, typically leading to a supersymmetric anti de Sitter (AdS) geometry in 4D. In the large volume scenario [38], instead, they are stabilized using an interplay of $\alpha^{\prime}$ corrections with nonperturbative corrections, such that the $4 \mathrm{D}$ vacuum is also AdS but nonsupersymmetric. In what follows we will mainly focus on the former approach, the only exception being Sec. V, where we will combine $\alpha^{\prime}$ corrections with a gaugino condensate to stabilize the Kähler modulus.

(iii) The final step consists of uplifting the 4D geometry to de Sitter space. Many proposals exist to achieve this goal (see e.g. [39-48]), the most common one being the inclusion of a supersymmetry-breaking source, most prominently an $\overline{D 3}$-brane, at the bottom of a warped throat [13].

These steps are usually followed through entirely within the framework of 4D supergravity, the low energy effective field theory describing the 4D fields after the compactification. In this section we will quickly review some of its aspects.

Before doing so, let us give the main point of this section right away: the first two steps can be followed with reasonable amount of control purely within the 4D effective field theory. However, in many models of moduli stabilization [13] the uplift cannot be parametrically decoupled from Kähler moduli stabilization. In these cases, although we believe that there is nothing wrong with the use of 4D supergravity as a matter of principle, the question whether or not a proposed uplift can be successful heavily depends on the detailed moduli dependence of the uplift ingredient. We will argue that at least for $\overline{D 3}$-brane uplifts the required knowledge about the moduli dependence is hard to acquire from 4D effective field theory reasoning alone. In other words, guessing the correct effective field theory is not as obvious as it might seem.

\section{A. Complex structure moduli stabilization}

The effective 4D supergravity that governs the light moduli of type IIB 03/O7 CY-orientifolds is well known. For a single Kähler modulus (i.e. $h_{1,1}^{+}=1$ ) the Kähler potential is given by

$$
\begin{aligned}
\mathcal{K}\left(\rho, z_{\alpha}, \tau\right)= & -3 \ln (-i(\rho-\bar{\rho}))-\ln (-i(\tau-\bar{\tau})) \\
& -\ln \left(-i \int_{M_{6}} \Omega \wedge \bar{\Omega}\right)
\end{aligned}
$$

to leading order in the $\alpha^{\prime}$ expansion. Since we are considering a unique Kähler modulus $\rho$, this is the (complexified) volume modulus $\left[\operatorname{Im}(\rho)=\mathcal{V}^{2 / 3}\right]$. Also, $\tau=C+i e^{-\phi}$ is the axiodilaton and $z_{\alpha}$ are the $h_{2,1}^{-}$complex structure moduli that appear in the Kähler potential implicitly via the holomorphic three-form $\Omega=\Omega\left(z_{\alpha}\right)$.

Dilute three-form fluxes (such that warping effects are negligible) generate a superpotential, the Gukov-VafaWitten (GVW) superpotential [49]

$$
W_{\mathrm{GVW}}=\sqrt{\frac{2}{\pi}} \int_{M_{6}} G_{3} \wedge \Omega,
$$

where $G_{3}=F_{3}-\tau H_{3}$ is the complexified three-form flux. This superpotential depends holomorphically on the axiodilaton and the complex structure moduli. It was derived by comparing the tension $\mathcal{T}^{D W}$ of Bogomol'nyi-PrasadSommerfield (BPS) domain walls that separate flux vacua with different three-form fluxes (i.e. D5/NS5-branes that wrap internal three-cycles) with the change in superpotential $\Delta W$ across the domain wall and demand the standard 4D SUSY relation

$$
\mathcal{T}^{D W}=2 e^{\mathcal{K} / 2}|\Delta W| .
$$

The SUSY conditions $D_{i} W=0$ (where $i$ runs over the complex structure moduli and the axiodilaton) require that $G_{3}$ is of Hodge type $(2,1) \oplus(0,3)$. This should be read as an equation for the complex structure moduli and the axiodilaton, which obtain masses of the order

$$
m_{C S} \sim \frac{\alpha^{\prime}}{R^{3}},
$$

where $R$ is a typical scale of the internal geometry. Thus, at large volume they are much lighter than the Kaluza-Klein (KK) modes of the internal geometry. If the superpotential is entirely determined by the GVW superpotential, which does not depend on the Kähler modulus $\rho$, the 4D potential vanishes at the minimum of the complex structure moduli and the axiodilaton due to the no-scale relation $\mathcal{K}^{\rho \bar{\rho}} \partial_{\rho} \mathcal{K} \partial_{\bar{\rho}} \mathcal{K}=3$. This means that the volume modulus remains massless and the final SUSY condition

$$
0=D_{\rho} W=\left(\partial_{\rho} \mathcal{K}\right) W
$$

cannot be solved at finite volume unless the nongeneric condition $W=0$ happens to be met, i.e. $G_{3}$ is of Hodge type $(2,1)$.

The upshot is that for generic fluxes all but the single Kähler modulus are stabilized and SUSY is broken. Hence, at low energies one is left with a single modulus $\rho$ and a constant superpotential $W=W_{0}$.

\section{B. Kähler moduli stabilization: KKLT}

In order to stabilize the volume modulus, KKLT argued for a $\rho$-dependent correction of the superpotential [13]. Because the superpotential must depend holomorphically 
on the volume modulus and the real part of $\rho$ enjoys a continuous shift symmetry to all orders in perturbation theory, the only possible corrections to the superpotential are nonperturbative

$$
\delta W_{\mathrm{np}}=\sum_{n} A_{n} e^{i a_{n} \rho},
$$

and break the continuous shift symmetry of $\operatorname{Re}(\rho)$ to a discrete one. There are two kinds of nonperturbative effects that can generate the desired exponential terms:

(a) Euclidean D3-branes wrapping internal fourcycles [50].

(b) Gaugino condensation on a stack of $N D 7$-branes [17-20].

In what follows we will focus on (b): a stack of $N D 7$ branes wrapping a rigid four-cycle $\Sigma$, i.e. the position moduli of the D7's are massive. Assuming that no further branes intersect the 7-brane stack (i.e. light flavors are absent) the low energy effective theory is $S U(N)$ SUSY Yang-Mills (YM). At low energies this theory confines and develops a nonperturbative superpotential via gaugino condensation,

$\langle\lambda \lambda\rangle=-32 \pi^{2} \Lambda^{3}, \quad \Rightarrow \delta W_{\mathrm{np}}=-\frac{N}{32 \pi^{2}}\langle\lambda \lambda\rangle=N \Lambda^{3}$,

where $\Lambda$ is the dynamical scale of the gauge theory which determines the perturbative running of the holomorphic gauge coupling $\tau_{\mathrm{YM}}=\frac{\Theta_{\mathrm{YM}}}{2 \pi}+i \frac{4 \pi}{g_{\mathrm{YM}}^{2}}$ via

$$
\tau_{\mathrm{YM}}=\frac{3 N}{2 \pi i} \ln \left(\frac{\Lambda}{\mu}\right), \quad \text { i.e. } \quad \Lambda=\mu e^{\frac{2 \pi i}{3 N} \tau_{\mathrm{YM}}} .
$$

By dimensionally reducing the $D 7$-brane action to $4 \mathrm{D}$, one can infer that classically the holomorphic gauge coupling is identified with the Kähler modulus $\rho$. In the quantum theory this equation should be read as the renormalization condition that matches the low energy effective theory to its UV completion. In other words

$$
\Lambda=\mu_{0} e^{\frac{2 \pi i}{N} \rho},
$$

where $\mu_{0}$ is a high scale. In summary, there is a nonperturbative correction of the superpotential

$$
\delta W_{\mathrm{np}}(\rho)=N \mu_{0}^{3} e^{\frac{2 \pi i}{N} \rho}
$$

that we may associate to a nonvanishing expectation value of the gaugino condensate $\langle\lambda \lambda\rangle$. Combining this with the constant flux superpotential $W_{0}$, at energies below the nonperturbative scale of the gauge theory the Kähler and superpotential read

$$
\mathcal{K}=-3 \ln (-i(\rho-\bar{\rho})), \quad W=W_{0}+A e^{i a \rho} .
$$

Now the $F$-term equation $D_{\rho} W=0$ is solved by

$$
W_{0}=-\left(1+\frac{2 a \operatorname{Im}(\rho)}{3}\right) A e^{i a \rho},
$$

and at the minimum of the potential the value of the vacuum energy is negative,

$$
V=-3 e^{\mathcal{K}}|W|^{2}=-\frac{a^{2}|A|^{2}}{12 \operatorname{Im} \rho} e^{-2 a \operatorname{Im} \rho}<0 .
$$

Control over the instanton expansion requires $a \rho>0$ and control over the $\alpha^{\prime}$ expansion of string theory requires $\rho \gg 0$. Both can be satisfied if due to a sufficient tuning of the flux configuration $\left|W_{0}\right| \ll 1$.

An essential feature of this stabilization mechanism is that the value of the cosmological constant and the mass of the volume modulus are related via

$$
\left|V_{\mathrm{AdS}}\right| \sim M_{\mathrm{P}}^{2} m_{\rho}^{2} .
$$

\section{The uplift}

The perhaps best understood way to uplift SUSY AdS vacua of type IIB string theory to de Sitter is by the inclusion of an anti-D3-brane at the bottom of a warped throat. This way of uplifting is attractive because in ISD flux backgrounds the antibrane is driven to strongly warped regions where its associated potential energy is naturally small [16]. Moreover such SUSY-breaking vacua are known to be connected to the SUSY ISD vacua via the nonperturbative KPV transition [16]. Arguably, no perturbative decay channel exists [35]. In this paper we assume perturbative stability of such a configuration.

Because the 10D warped throat supergravity solution is dual to the Klebanov-Strassler (KS) gauge theory [51], one should be able to equivalently describe the antibrane as a state of the KS gauge theory that breaks supersymmetry spontaneously rather than explicitly [35]. If this is the case, it has been argued that at very low energies the only degrees of freedom are the nilpotent goldstino multiplet $S$ (nilpotency means $S^{2}=0$, for constrained superfields; see e.g. [52]) and the volume modulus $\rho$ [53-61]. The proposals for the Kähler- and the superpotential were

$$
\begin{aligned}
& K=-3 \ln (-i(\rho-\bar{\rho})-S \bar{S}) \quad \text { and } \\
& W=W_{0}+A e^{i a \rho}+e^{2 \mathcal{A}_{0}} \mu^{2} S .
\end{aligned}
$$

Here, $\exp \left(2 \mathcal{A}_{0}\right) \mu^{2}$ parametrizes the strength of supersymmetry breaking with $\exp \left(\mathcal{A}_{0}\right)$ being the warp factor at the tip of throat, while $\mu$ is related to the unwarped tension of the anti-D3-brane as $|\mu|^{4} \sim T_{3}$.

In deriving the scalar potential one should treat $S$ as a usual chiral multiplet and in the end set $S=0$. For real parameters $W_{0}$ and $A$, the scalar potential reads 


$$
\begin{aligned}
V(\rho)= & \frac{a A e^{-a \operatorname{Im}(\rho)}}{6 \operatorname{Im}(\rho)^{2}}\left[A e^{-a \operatorname{Im}(\rho)}(a \operatorname{Im}(\rho)+3)\right. \\
& \left.+3 W_{0} \cos (a \operatorname{Re}(\rho))\right]+e^{4 \mathcal{A}_{0}} \frac{|\mu|^{4}}{12 \operatorname{Im}(\rho)^{2}} .
\end{aligned}
$$

The expectation of this being the correct way to describe the antibrane state comes from the fact that in the limit of vanishing nonperturbative stabilization $A \rightarrow 0$ one recovers the known runaway potential that is easily read off of the antibrane Dirac-Born-Infeld (DBI) and Chern-Simons (CS) action, while in the limit $\mu \rightarrow 0$ one recovers the supersymmetric KKLT potential. The corresponding potential is simply the sum of the (would-be) runaway $\overline{D 3}$ potential and the (would-be) supersymmetric KKLT potential. Tuning the tension contribution of the antibrane (by tuning the fluxes that determine the warp factor $e^{\mathcal{A}_{0}}$ ), one can obtain de Sitter vacua with tunably small cosmological constant [13]. Interestingly, within this description of the $\overline{D 3}$ induced uplift, the potential energy of the $\overline{D 3}$ adds on top of the negative potential at the supersymmetric AdS minimum to good approximation until a maximum uplift of about $\delta V \sim 2 \times\left|V_{\text {AdS }}\right|$. Beyond that we encounter runaway behavior $\rho \rightarrow \infty$.

However, we would like to point out that matching the $\mathrm{KPV}$ form of the $\overline{D 3}$-induced uplift does not uniquely determine the form of the superpotential. The route determining the dependence of $W$ on $S$ described above constitutes only one possible outcome of matching KPV. The ambiguity arises from the 4D point of view by the appearance of a new mass scale due to nonperturbative Kähler moduli stabilization, which was absent in the reduction leading to the KPV form of the $\overline{D 3}$-induced scalar potential. To see this in detail, we shall repeat the steps needed to match a candidate form of $W=W(S)$ to the KPV form of the uplift:

(i) The classical potential for an antibrane is determined by dimensional reduction over a classical no-scale Giddings, Kachru, and Polchinski (GKP) background [16].

(ii) The SUSY-breaking antibrane can be described within the 4D effective supergravity of volume stabilization in the KKLT setup by a nilpotent multiplet $S$ which incorporates the nonlinear SUSY of the antibrane into the supergravity description.

(iii) The $4 \mathrm{D}$ volume stabilization generates a new scale $m_{\rho}^{2} M_{\mathrm{P}}^{2}$.

(iv) In this situation, the classical potential of the antibrane carries over from the no-scale GKP background only in the limit $\delta V \gg m_{\rho}^{2} M_{P}^{2}$ (i.e. comparatively weak warping) while a constant uplift potential is appropriate in the limit $\delta V \ll m_{\rho}^{2} M_{P}^{2}$ where backreaction on the volume modulus can be neglected. (v) Matching with the no-scale reduction does not uniquely fix where the nilpotent superfield $S$ appears in the superpotential $W(S, \rho)$.

(vi) The sign of the vacuum energy as a function of increasing uplifting depends on the detailed form of $W(S, \rho)$.

The most general superpotential to leading order in the (fractional) instanton expansion is

$$
W=W_{0}+b \cdot S+A(1+c \cdot S) e^{i a \rho} .
$$

The special choice $c=0, b=e^{2 \mathcal{A}_{0}} \mu^{2}$ corresponds to the standard KKLT potential. The other extreme case would be to set $b=0$. In this case the SUSY KKLT vacuum corresponding to $c=0$ cannot be uplifted to de Sitter for any value of $c$. Obviously there exist infinitely many intermediate situations.. ${ }^{1}$

Of course it would be rather surprising if the term proportional to $c$ were completely absent for the following reason. It is well known that if mobile D3-branes are present, the coefficient of $e^{i a \rho}$ in the superpotential is a holomorphic function of their position moduli $[64,65]$. Their moduli space is therefore lifted by the same nonperturbative effects that lead to volume stabilization. If $D 3$-branes modify the coefficient of the exponential term in the superpotential, one would expect an $\overline{D 3}$-brane to do so as well.

However, this modification has to be quite drastic in order to have any important effect. In other words, because the coefficient $b$ is associated with the squared warp factor at the tip of the throat $e^{2 \mathcal{A}_{0}} \ll 1$ and the coefficient $c$ is multiplied by the gaugino condensate, the effect of the term $S e^{i a \rho}$ will be negligible if it is further suppressed by warping. At first glance this seems unreasonable as the antibrane sits in a strongly warped region of space-time. So, why should there exist any unwarped contributions to the superpotential? First of all, there is no 4D EFT reason that would forbid such a term so in the spirit of a Wilsonian effective action we should include it. If coefficients in the effective action are small, there should better exist a good reason for their smallness. We have such a reason, namely warping, only for the classical term. On the contrary, we have no a priori reason to assume smallness also of the $S$ coupling to the nonperturbative term. Still, an unsuppressed coupling of the $S$ field parametrizing a warped anti-D3brane may sound quite counterintuitive. While we must

\footnotetext{
${ }^{1}$ Note that this is not the most general way to parametrize the antibrane potential (see e.g. [62]). More generally, starting from Eq. (15) one may rescale $S \rightarrow e^{-2 \mathcal{A}_{0}} S$ such that the warp factor appears in the Kähler rather than in the superpotential as in [63]. The leading nonperturbative corrections to the antibrane potential could then be encoded by replacing $e^{4 \mathcal{A}_{0}} \rightarrow e^{4 \mathcal{A}_{0}}+\alpha(\rho, \bar{\rho})$. Only for special choices of the real function $\alpha(\rho, \bar{\rho})$ this can be transformed into a superpotential correction. We thank Susha Parameswaran for bringing this to our attention.
} 
leave a fuller discussion to later when we have introduced the full 10D description, we note here that the presence of the nonperturbative term signals the presence of a new mass scale $\sim\langle\lambda \lambda\rangle$ in the effective physics. Absent any protective symmetries or dynamical coupling suppression, such a new mass scale will communicate itself generically to all other scalar couplings, in particular the warped KK scale. This would in turn lead one to naively expect couplings between $S$ and $\langle\lambda \lambda\rangle$ of $\mathcal{O}(1)$ following the spirit of Wilsonian EFT.

Given this, to us it appears plausible that any energy density, be it in a warped throat or in the bulk Calabi-Yau, effectively couples to the nonperturbative effects without any further warp. Hence, to us the following superpotential seems to adequately parametrize the interplay of an antibrane with nonperturbative volume stabilization,

$$
W=W_{0}+e^{2 \mathcal{A}_{0}} \cdot \mu^{2} \cdot S+A\left(1+c\left(\mu^{2}\right) \cdot S\right) e^{i a \rho} .
$$

Again, $|\mu|^{4}$ is associated with the unwarped tension of the antibrane. $e^{2 \mathcal{A}_{0}}$ is the IR warp factor of the classical ISD background while $c\left(\mu^{2}\right)$ is an unknown function of the antibrane tension that we believe is not suppressed by powers of the warp factor. Whether or not an uplift to de Sitter is possible depends on the magnitude and functional form of $c\left(\mu^{2}\right) .^{2}$

The observation that the fate of the uplift depends so heavily on the details of the moduli potential can be embedded into a more systematic approach towards a de Sitter uplift: in the 4D effective field theory one may expand the scalar potential (before any attempt to uplift it) around its SUSY AdS (or Minkowski) minimum,

$$
V=V_{0}+\sum_{i} \frac{1}{2} m_{i}^{2} u_{i}^{2}+\cdots
$$

with squared masses $m_{i}^{2}$ (assuming canonical normalization).

Including an uplift means adding a further term $\delta V\left(u_{i}\right)$ to the potential,

$$
V\left(u_{i}\right) \rightarrow \tilde{V}\left(u_{i}\right)=V\left(u_{i}\right)+\delta V\left(u_{i}\right)
$$

Although in general in order to determine the value of the potential at the new minimum $u_{i}^{1}$ one has to minimize the new potential $\tilde{V}$ with respect to all moduli $u_{i}$ one easily sees that the resulting shift in potential energy can be expanded in powers of $\delta V$ as

\footnotetext{
${ }^{2}$ We consider an uplift to de Sitter successful if it does not reduce the mass scale of the lightest modulus as compared to the pre-uplifted configuration by factors that control the perturbative expansion of string theory such as cycle volumes or the dilaton. Only with this restriction, a truncation to the lowest order Kähler potential necessary for moduli stabilization is consistent in presence of the uplift.
}

$$
\begin{aligned}
\tilde{V}\left(u_{i}^{1}\right)-V\left(u_{i}^{0}\right)= & \delta V\left(u_{i}^{0}\right)\left(1-\sum_{i} \frac{1}{2} \frac{\left(\left.M_{\mathrm{P}} \partial_{u_{i}} \delta V\right|_{u_{i}^{0}}\right)^{2}}{M_{\mathrm{P}}^{2} m_{i}^{2} \delta V\left(u_{i}^{0}\right)}\right. \\
& \left.+\mathcal{O}\left(\left(\delta V / m_{i}^{2} M_{\mathrm{P}}^{2}\right)^{2}\right)\right) .
\end{aligned}
$$

Evidently, for sufficiently small uplift potential $\delta V$ the backreaction on the fields $u^{i}$ can be neglected and the extra potential energy simply adds up to the preexisting one. However, in the simplest examples of moduli stabilization [13] the value of the cosmological constant at the supersymmetric minimum $V_{\text {AdS }}$ is tied to the mass-scale $m_{\rho}$ of the lightest modulus

$$
\left|V_{\mathrm{AdS}}\right| \sim m_{\rho}^{2} M_{\mathrm{P}}^{2}
$$

Clearly, in order to uplift such vacua to de Sitter, an uplift potential $\delta V$ has to be added that is at least of the order of $\left|V_{\text {AdS }}\right|$. In this case the expansion in (21) is not under parametric control and the strength of backreaction effects heavily depends on the detailed moduli dependence of $\delta V$. For the uplifted KKLT potential a strong backreaction effect does not occur due to the proposed power-law dependence of $\delta V \equiv\left|\mu^{4}\right| / 12 \operatorname{Im}(\rho)^{2}$. In this case the first correction in (21) is suppressed by a volume factor $(a \operatorname{Im}(\rho))^{-2} \ll 1$. In contrast, for an exponential dependence $\delta V \sim e^{-2 a \operatorname{Im}(\rho)}$ corrections in (21) are unsuppressed. This is precisely what a sufficiently dominant term $\propto c\left(\mu^{2}\right)$ in (18) would entail.

We conclude that if the vacuum potential of the SUSY $\mathrm{AdS}_{4}$ vacua can be parametrically decoupled from the mass scale of the lightest modulus,

$$
\left|V_{\text {AdS }}\right| \ll m_{\rho}^{2} M_{\mathrm{P}}^{2}
$$

the required uplift can be small enough such that backreaction effects are negligible and thus, de Sitter uplifts are generically possible. Conversely, if this is not the case, detailed knowledge about the moduli dependence of $\delta V\left(u_{i}\right)$ is required in order to determine the uplift in potential energy beyond parametric estimates.

Finally, we summarize the upshot of this section and set the goal for the rest of the paper:

(i) In order to determine if an antibrane can uplift the SUSY KKLT vacua to de Sitter we need more information about the interplay of the antibrane with nonperturbative effects.

(ii) In the remainder of the paper we will determine only the sign of the cosmological constant in the presence of an antibrane uplift using a 10D description. This will allow us to constrain the coefficients of $W(S, \rho)$ only to the extent that the sign of the $4 \mathrm{D}$ vacuum energy as determined from $W(S, \rho)$ must be matched to the $10 \mathrm{D}$ result. In particular we will not determine the form of the scalar potential $V(\rho)$ away from its 
minimum. This important task that would uniquely fix the coefficients in the superpotential we leave for future work.

\section{SOME HIGHER-DIMENSIONAL CONSIDERATIONS}

In view of the surprising difficulties that one usually encounters when trying to construct consistent de Sitter vacua in string theory, we find it worthwhile to investigate if the seemingly conspirative modification of the 4D effective field theory that would prevent an uplift to de Sitter indeed occurs.

Clearly the cleanest way to do this would be to derive the correct effective field theory of the volume modulus together with all its SUSY-breaking states from first principles. Due to the obvious difficulty of this approach, we will opt for another one. Instead of deriving the off-shell $4 \mathrm{D}$ effective potential, we will constrain it using a 10D onshell description of the nonperturbative effects as well as the uplift. Before turning to the 10D setup we study some general aspects of compactifications that will later be relevant and use them to explain this notion of an on-shell description. This concept will be clarified in terms of a toy model that will also be useful to develop a physical intuition that is applicable to the case of type IIB string theory.

\section{A. On-shell vs off-shell potentials}

Let us first explain what we mean by on-shell and offshell potentials. We refer to the usual $4 \mathrm{D}$ potential that is determined by straightforward dimensional reduction as the off-shell potential. It is easily determined from the higherdimensional Einstein equations as follows: for a theory of $D=d+4$ dimensional gravity and further fields compactified to 4D de Sitter, Minkowski, or anti de Sitter, the most general $D$-dimensional metric ansatz is

$$
d s^{2}=e^{2 \mathcal{A}(y)} \tilde{g}_{\mu \nu}^{4}(x) d x^{\mu} d x^{n u}+g_{m n}^{d}(y) d y^{m} d y^{n},
$$

with warp factor $e^{\mathcal{A}}, 4 \mathrm{D}$ coordinates $x^{\mu}$, and internal coordinates $y^{m}$. The higher-dimensional Einstein equations

$$
R_{M N}-\frac{1}{2} g_{M N} R=T_{M N}
$$

determine the effective 4D potential in terms of the internal curvature $R_{d}$ and the higher-dimensional energymomentum tensor $T_{M N}$,

$$
\begin{aligned}
V \cdot M_{\mathrm{P}}^{-4} & =\frac{1}{4} \tilde{R}_{4} \cdot M_{P}^{-2} \\
& =\mathcal{V}_{w}^{-2} M^{d} \int d^{d} y \sqrt{g^{d}} e^{4 \mathcal{A}} \frac{\left(-\frac{1}{4} T_{\mu}^{\mu}-\frac{1}{2} R_{d}\right)}{M^{2}},
\end{aligned}
$$

where $M$ is the $D$-dimensional Planck mass, $\mathcal{V}_{w}=$ $M^{d} \int d^{d} y \sqrt{g^{d}} e^{2 \mathcal{A}}$ is the warped volume, and $M_{\mathrm{P}}^{2}=$ $M^{2} \mathcal{V}_{w}$ is the 4D Planck mass. 4D vacua of the higherdimensional theory correspond to local minima of this potential which encodes the value of the cosmological constant as well as the scalar mass spectrum.

In many instances it is hard to determine this potential in full explicitness and it may be very useful to use an alternative expression for the 4D potential that is only valid when all geometric moduli are assumed to be at their minimum. One may obtain such an expression by considering the trace-reversed Einstein equations that relate the internal space Ricci scalar $R_{d}$ to the energy-momentum tensor. The result is

$V \cdot M_{\mathrm{P}}^{-4}=\mathcal{V}_{w}^{-2} M^{d} \int d^{d} y \sqrt{g^{6}} e^{4 \mathcal{A}} \frac{(D-6) T_{\mu}^{\mu}-4 T_{m}^{m}}{4(D-2) M^{2}}$.

It is important to realize that in writing this expression one has integrated out all geometric moduli, that is the potential is only valid at the minimum of the off-shell potential (26) where the two coincide [66]. This has several consequences: for example, if a higher-dimensional source contributes an energy-momentum tensor $\delta T_{M N}$ with

$$
(D-6) \delta T_{\mu}^{\mu}-4 \delta T_{m}^{m}<0,
$$

one must not conclude that the 4D cosmological constant decreases when the source is included since all other terms in the full energy-momentum tensor $T_{M N}$ will be perturbed even to leading order in $\delta T_{M N}$. Although this means that any on-shell potential has to be treated carefully, one can derive powerful statements from it. For example, in the absence of any source satisfying

$$
(D-6) T_{\mu}^{\mu}-4 T_{m}^{m} \geq 0,
$$

the $4 \mathrm{D}$ vacuum energy can never be positive. Using this, one may show that if one only allows for $p$-form fluxes with $1 \leq p \leq D-1$ and localized objects of positive tension and codimension $\geq 2$, de Sitter solutions are ruled out [67]. Further details are given in Appendix B.

This means that in many interesting examples the onshell potential encodes subtle backreaction effects that correspond to the correction terms in (21): whenever a source of positive higher-dimensional energy is turned on that does not satisfy the condition (29) in a compactification that is stabilized by sources that also do not satisfy the condition, higher-order corrections in (21) conspire to keep the overall potential energy negative.

\section{B. A simple example: Freund-Rubin compactification}

We will now demonstrate this behavior using the wellknown Freund-Rubin compactification. This is a 6D theory 
compactified on a $S^{2}$, with the $S^{2}$ stabilized by two-form fluxes [68]. The $6 \mathrm{D}$ action is

$$
S_{6}=\frac{M^{4}}{2} \int\left(* R_{6}-\frac{1}{2} F_{2} \wedge * F_{2}\right),
$$

with a two-form field-strength $F_{2}=d A_{1}$. The equations of motion/Bianchi identity are

$$
R_{M N}=\frac{1}{2} F_{M P} F_{N}^{P}-\frac{1}{8} g_{M N}\left|F_{2}\right|^{2}, \quad d F_{2}=0=d * F_{2} .
$$

These admit a solution where the $6 \mathrm{D}$ geometry is a product $\mathrm{AdS}_{4} \times S^{2}$ and the $S^{2}$ is threaded by $N$ units of two-form flux $F_{2}=\frac{N}{2 q} \omega_{2}$. Here, $\omega_{2}$ is the volume form of the $S^{2}$ normalized to $\int_{S^{2}} \omega_{2}=4 \pi$ and $q$ is the $U(1)$ charge of the particle that couples electrically to $A_{1}$ with smallest charge. The $S^{2}$ radius is fixed at

$$
L_{0}^{2}=\frac{3 N^{2}}{32 q^{2}},
$$

and in agreement with (27) the 4D vacuum energy reads

$$
V \cdot M_{\mathrm{P}}^{-4}=\mathcal{V}^{-2} M^{2} \int d^{2} y \sqrt{g^{S^{2}}} \frac{\left(-2\left|F_{2}\right|^{2}\right)}{16 M^{2}}=-\left(12 \pi M^{4} L_{0}^{4}\right)^{-1} .
$$

One may try to uplift the four-dimensional vacuum energy by adding a number $N_{3}$ of three-branes of positive tension $T_{3}$ smeared over the internal two-sphere. Clearly they are a source of energy density and therefore the expectation is that they give rise to an increase of the four-dimensional vacuum energy. However, their energy-momentum tensor satisfies $(D-6) T_{\mu}^{\mu}-4 T_{m}^{m}=0$ and so there is no new contribution under the integral in (26). Even without knowing the full solution to the $6 \mathrm{D}$ equations of motion, we can hence express the $4 \mathrm{D}$ vacuum energy as a function of the a priori unknown size of the two-sphere:

$$
V \cdot M_{\mathrm{P}}^{-4}=\mathcal{V}^{-2} M^{2} \int d^{2} y \sqrt{g^{S^{2}}} \frac{\left(-2\left|F_{2}\right|^{2}\right)}{16 M^{2}}=-\frac{M^{2} L_{0}^{2}}{12 \pi M^{6} L_{1}^{6}},
$$

where $L_{0}$ is given in Eq. (32) and $L_{1}$ is the adjusted length scale of the two-sphere. Here, the wonders of on-shell potentials manifest themselves explicitly for the first time: The source of uplift in potential energy, the three-brane tension, has apparently disappeared from the integrand of the on-shell potential. It does, however, appear implicitly through the dependence of the $S^{2}$ radius on the three-brane tension. This way of parametrizing the $4 \mathrm{D}$ vacuum energy in terms of a single unknown quantity (in this case the radius) should be kept in mind as it will play an important role in the remainder of the paper. From Eq. (34) one may already follow that no matter how much three-brane tension is added, the vacuum energy cannot increase beyond zero but at most approaches zero from below. In this simple 6D case one can do better and from the internal Einstein equations determine $L_{1}$ as a function of the three-brane tension:

$$
L_{1}^{2}=\left(1-\mathcal{T}_{3}\right)^{-1} L_{0}^{2}, \quad \text { with } \quad \mathcal{T}_{3} \equiv \frac{N_{3} T_{3}}{4 \pi M^{4}}
$$

Plugging this into Eq. (34) we see that the vacuum energy adjusts and as expected to leading order in the dimensionless tension $\mathcal{T}_{3}$ the change in potential energy corresponds precisely to the three-brane tension

$$
\delta\left(V \cdot M_{\mathrm{P}}^{-4}\right)=\frac{\mathcal{T}_{3}}{4 \pi L_{0}^{4} M^{4}}+\mathcal{O}\left(\mathcal{T}_{3}^{2}\right)=\frac{N_{3} T_{3}}{M_{\mathrm{P}}^{4}}+\mathcal{O}\left(\mathcal{T}_{3}^{2}\right)
$$

Of course, as we increase the three-brane tension such that $\mathcal{T}_{3} \rightarrow 1$ the $4 \mathrm{D}$ vacuum energy increases, but the higherorder terms become relevant and will prevent an uplift to de Sitter. Therefore, the 4D vacuum energy will approach zero from below, the limit being $\mathcal{T}_{3}=1$, where the $S^{2}$ decompactifies. Note that as predicted by (21) the expansion parameter that controls backreaction is given by $\delta V / m_{\mathrm{KK}}^{2} M_{\mathrm{P}}^{2}$ because the $\mathrm{KK}$ scale is the mass of the lightest modulus.

One might be concerned that three-branes and two-form fluxes share an intrinsic property making them unsuitable uplifting ingredients because they appear with the wrong sign under the integral of (27). Of course, if one only includes these ingredients in the compactification, de Sitter solutions are ruled out [67]. But as we now demonstrate it is enough to include also a positive $6 \mathrm{D}$ c.c., or equivalently a five-brane of tension $T_{5} \equiv \mathcal{T}_{5} M^{6}$, for an uplift to de Sitter by three-branes or fluxes to be possible (see also [40] for related conclusions). In this case the size of the two-sphere is bounded from above via

$$
l^{2} \equiv L^{2} M^{2}=\frac{1-\mathcal{T}_{3}}{\mathcal{T}_{5}}\left(1-\sqrt{1-\frac{3}{16} \frac{\mathcal{T}_{5} n^{2}}{\left(1-\mathcal{T}_{3}\right)^{2}}}\right) \leq \frac{1-\mathcal{T}_{3}}{\mathcal{T}_{5}},
$$

where $n \equiv N \cdot M / q$ corresponds to the number of two-form flux quanta. When $n^{2}>n_{\max }^{2} \equiv \frac{16}{3} \mathcal{T}_{5}^{-1}\left(1-\mathcal{T}_{3}\right)^{2}$ the sphere decompactifies. Thus, in order for the curvature of the sphere to be sub-Planckian we need both a small positive $6 \mathrm{D}$ cosmological constant $\mathcal{T}_{5} \ll 1$ as well as a large number of two-form fluxes $n$. 

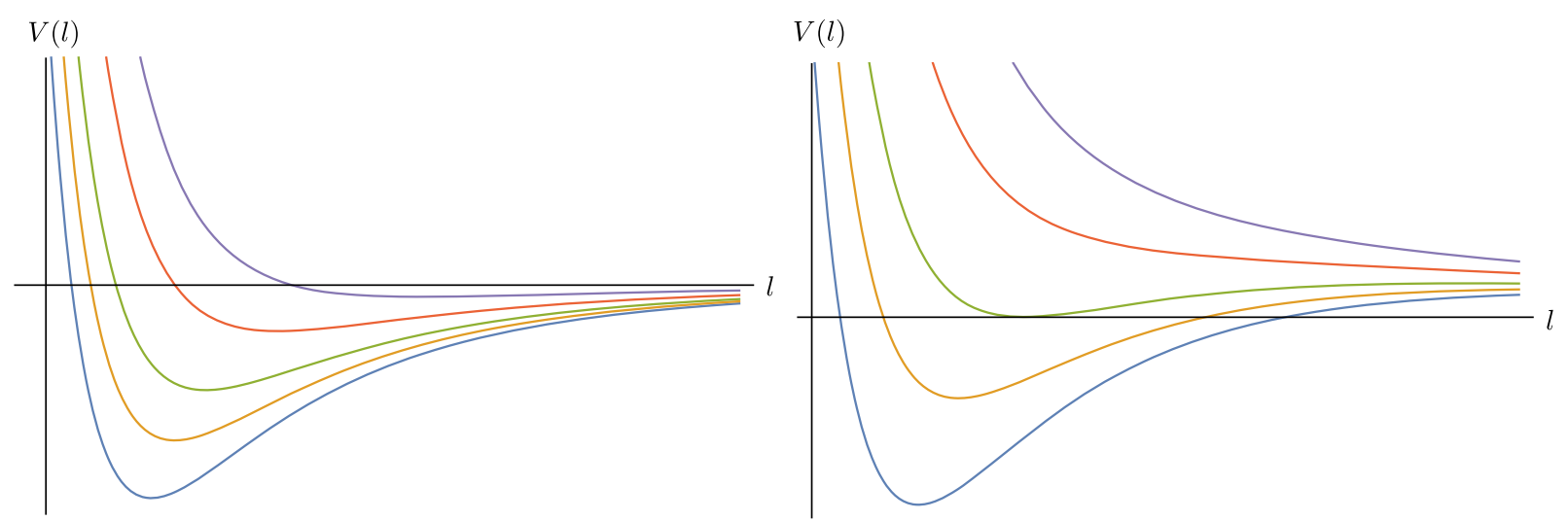

FIG. 1. Left: off-shell potential $V(l)$ for the $S^{2}$ volume modulus in the case without a $6 \mathrm{D}$ c.c. for $n=25$ flux units and different values of the dimensionless three-brane tension $\mathcal{T}_{3}: \mathcal{T}_{3}=0$ in blue, $\mathcal{T}_{3}=0.1$ in yellow, $\mathcal{T}_{3}=0.21$ in green, $\mathcal{T}_{3}=0.4$ in red, and $\mathcal{T}_{3}=0.6$ in purple. As can be seen, the more energy density, the higher the vacuum energy, but the flattening prevents the minimum to go above zero. Right: off-shell potential $V(l)$ for the $S^{2}$ volume modulus in the case with a $6 \mathrm{D}$ c.c. for $n=25$ flux units, $\mathcal{T}_{5}=0.004$ and different values of the dimensionless three-brane tension $\mathcal{T}_{3}: \mathcal{T}_{3}=0$ in blue, $\mathcal{T}_{3}=0.1$ in yellow, $\mathcal{T}_{3}=0.21$ in green, $\mathcal{T}_{3}=0.4$ in red, and $\mathcal{T}_{3}=0.6$ in purple. This time it is also possible to find de Sitter minima once enough three-brane tension has been added.

The on-shell potential according to (27) reads

$$
V \cdot M_{\mathrm{P}}^{-4}=\frac{1}{16 \pi}\left[\frac{2 \mathcal{T}_{5}}{l^{2}}-\frac{n^{2}}{8 l^{6}}\right]
$$

Clearly the no-go theorem of Maldacena and Nunez [67] is evaded and de Sitter vacua are possible although not generic. ${ }^{3}$ We give a concrete example in Fig. 1.

For completeness we also give the off-shell potential (plotted in Fig. 1)

$$
V \cdot M_{\mathrm{P}}^{-4}(l)=\frac{1}{16 \pi}\left[\frac{4 \mathcal{T}_{5}}{l^{2}}+\frac{n^{2}}{4 l^{6}}-\frac{4\left(1-\mathcal{T}_{3}\right)}{l^{4}}\right]
$$

Evidently, the flattening behavior observed for the case with only fluxes and three-branes does not exhibit any intrinsic feature of branes and fluxes but is merely a property of the simple scheme of moduli stabilization. By including a positive $6 \mathrm{D}$ c.c. it was possible to decouple the lightest modulus mass from the value of the $4 \mathrm{D}$ c.c. such that a small perturbation could uplift it to 4D de Sitter.

\footnotetext{
${ }^{3}$ As an aside we note that the de Sitter vacua with largest possible cosmological constant lie along the line of marginal stability $n=n_{\max }$ for which $l^{2}=l_{\max }^{2} \equiv \frac{1-\mathcal{T}_{3}}{\mathcal{T}_{5}}$. The value of the c.c. is given by$$
V_{\max } \cdot M_{\mathrm{P}}^{-4}=\frac{\mathcal{T}_{5}}{12 \pi} \frac{\mathcal{T}_{5}}{\underbrace{1-\mathcal{T}_{3}}_{=l_{\max }^{-2} \ll 1}} \lesssim l_{\max }^{-4} .
$$

It is of order the higher-dimensional c.c. times the usual volume suppression factor. Note, however, that the required tuning of the $6 \mathrm{D}$ c.c. amounts to a further volume suppression $V_{\max } \lesssim l^{-4}$.
}

\section{THE 10D PERSPECTIVE}

We would now like to study moduli stabilization and the uplift to de Sitter space from a ten-dimensional point of view. The classical part is well understood: the GukovVafa-Witten superpotential can be lifted to the tendimensional three-form potential of type IIB supergravity [49] and the 4D SUSY conditions that determine the threeform fluxes to be of Hodge-type $(2,1)$ lift to the 10D SUSY conditions of $B$ type [69]. Furthermore the 4D scalar potential is minimized precisely when the $10 \mathrm{D}$ equations of motion are solved by the imaginary self-dual solutions of [14].

An analogous 10D $\leftrightarrow 4 \mathrm{D}$ correspondence of Kähler moduli stabilization is somewhat harder to establish, both conceptually as well as technically ${ }^{4}$ : the dynamical origin of the exponential superpotential is the condensation of gaugino bilinears in the 4D SUSY YM gauge theory (or euclidean D3-brane instantons). The scale below which the condensation occurs is the dynamical scale of the gauge theory which typically lies far below the Kaluza-Klein scale. So, how can it be possible even in principle to include the nonperturbative effects in a higher-dimensional setup? First, there certainly exist geometrical setups compatible with the correct order of scales: an example is that of an "anisotropic" Calabi-Yau space in which the four-cycle that the 7-branes wrap is much smaller than the typical length scale of the transverse space [70]. In this case the nonperturbative scale of gaugino condensation can lie far below the Kaluza-Klein scale of the four-cycle and at the same scale as the transverse Kaluza-Klein scale. Another situation of this type corresponds to a compactification space

\footnotetext{
${ }^{4}$ We thank Arthur Hebecker for a very helpful discussion concerning this point.
} 
that is equipped with warped throats of significant warping. In this case the warped Kaluza-Klein scale lies exponentially below the bulk KK scale.

There has, however, been crucial progress in recent years in establishing a far more general ten-dimensional picture of gaugino condensation [65,71-73]. First, note that if a mobile D3-brane is present, the classical moduli space of the world-volume scalars is identified with the compactification geometry. In the absence of nonperturbative effects, there is no potential for the world-volume scalars and the internal geometry can thus be probed at arbitrarily small energies. Thus, even if nonperturbative effects generate a potential for the world-volume scalars one may probe the (quantum-deformed) internal geometry at scales that lie far below the KK scale. With this in mind one should be able to effectively describe the SUSY vacua with nonperturbative Kähler stabilization by the 10D equations of motion, corrected at order of the value of the gaugino condensate $\langle\lambda \lambda\rangle$.

So, how is the 10D supergravity corrected in order to account for the nonperturbative effects? Remarkably, the following simple prescription advocated by the authors of $[70,72,74]$ seems to capture at least the leading order effects:

(a) Use the classical type IIB supergravity together with the DBI and CS actions for localized objects to quadratic order in the world-volume fermions.

(b) Solve the 10D equations of motion, assuming that the fermion bilinear that corresponds to the 7-brane gaugino is nonvanishing.

Clearly this approach needs to be justified. For this let us consider the nonperturbative lifting of the $D 3$-brane position moduli space. This can be studied from different angles. The first is the standard 4D perspective. In compactifications with both $D 7$-branes and mobile $D 3$-branes the gauge-kinetic function of the D7-brane gauge theory depends on the open-string $D 3$-brane position moduli $z^{i}$ via one-loop open string threshold corrections which were calculated explicitly for a $T^{4} / \mathbb{Z}_{2} \times T^{2}$ orientifold of type IIB string theory [64]. Then, at low energies the nonperturbative superpotential is a function of the position moduli $z^{i}$ which obtain a potential. The open string calculation of [64] was perfectly matched with a dual closed string calculation in [65,71] as follows: a mobile $D 3$-brane treated as a classical localized source in the 10D supergravity induces corrections to the volume of the fourcycle that the D7-branes wrap which determines the gaugecoupling of the $D 7$ gauge theory. Again, the $D 3$ position moduli enter the nonperturbative superpotential in the 4D $\mathrm{EFT}^{5}$ The closed string computation is particularly useful as it readily generalizes beyond simple toroidal orientifolds. In particular, for a stack of $N$ D7-branes with holomorphic

\footnotetext{
${ }^{5}$ See also $[75,76]$ for a derivation using the language of generalized complex geometry.
}

embedding equation $h(z)=0$, the gauge-kinetic function $f\left(\rho, z^{i}\right)$ of the $D 7$ gauge theory depends on the volume modulus $\rho$ as well as the $D 3$ position moduli $z^{i}$ [65]

$$
f(\rho, z)=\rho+\frac{\ln h(z)}{2 \pi i} .
$$

Using this dependence of the gauge-kinetic function $f$ on the D3-brane position moduli, one may determine the $4 \mathrm{D}$ nonperturbative superpotential to be

$$
W \propto e^{\frac{2 \pi i}{N} f}=h(z)^{1 / N} e^{\frac{2 \pi i}{N} \rho} .
$$

So far, classical 10D physics has been used only to obtain the gauge kinetic function (41) while the generation of a nontrivial potential for the D3-brane moduli is determined entirely within 4D effective field theory. Crucially these two steps could be separated because the classical backreaction of a $D 3$-brane on the classical $10 \mathrm{D}$ supergravity solution is finite. This is clearly not the case for a $\overline{D 3}$-brane, so the quantum corrected $10 \mathrm{D}$ action is needed. So how can it be deduced? The key points were derived in [70], where the authors analyzed the generation of a nontrivial classical potential for the position moduli of D3-branes in ISD backgrounds subject to harmonic nonISD perturbations. Crucially, it was shown that in conifold backgrounds every superpotential that can be written down for the position moduli in the 4D effective field theory can be matched to a noncompact classical 10D supergravity solution such that the scalar potentials coincide. Hence, the quantum corrected 10D supergravity that reproduces the correct $D 3$-brane potential is only corrected by terms that are localized away from the warped throat. Such localized terms are necessary because the entirely uncorrected type IIB supergravity equations do not admit static non-ISD perturbations in the compact case due to the global constraints of [14].

It is tempting to identify these localized terms with the terms in the 7-brane action that are proportional to the gaugino bilinear $\langle\lambda \lambda\rangle$. Indeed, the superpotential (42) can be encoded in so-called series $I$ three-form flux

$$
\left(G_{3}\right)_{i \bar{j} \bar{k}} \propto\langle\lambda \lambda\rangle \nabla_{i} \nabla_{l} \operatorname{Re}(\ln h(z)) g^{l \bar{m}} \bar{\Omega}_{\bar{m} \bar{j} \bar{k}}
$$

where $\Omega$ is the holomorphic three-form of the Calabi-Yau [70]. This is precisely the perturbation of three-form fluxes that is sourced by the fermionic bilinear term in the D7-brane action (extrapolated to the non-Abelian case). Guided by this nontrivial consistency check we believe that the relevant details of nonperturbative volume stabilization are indeed captured by the classical 10D supergravity action assuming a nonvanishing expectation value of the gaugino bilinear. While (by construction) the 10D and 4D pictures can be used equivalently to determine the nonperturbative $D 3$-brane 


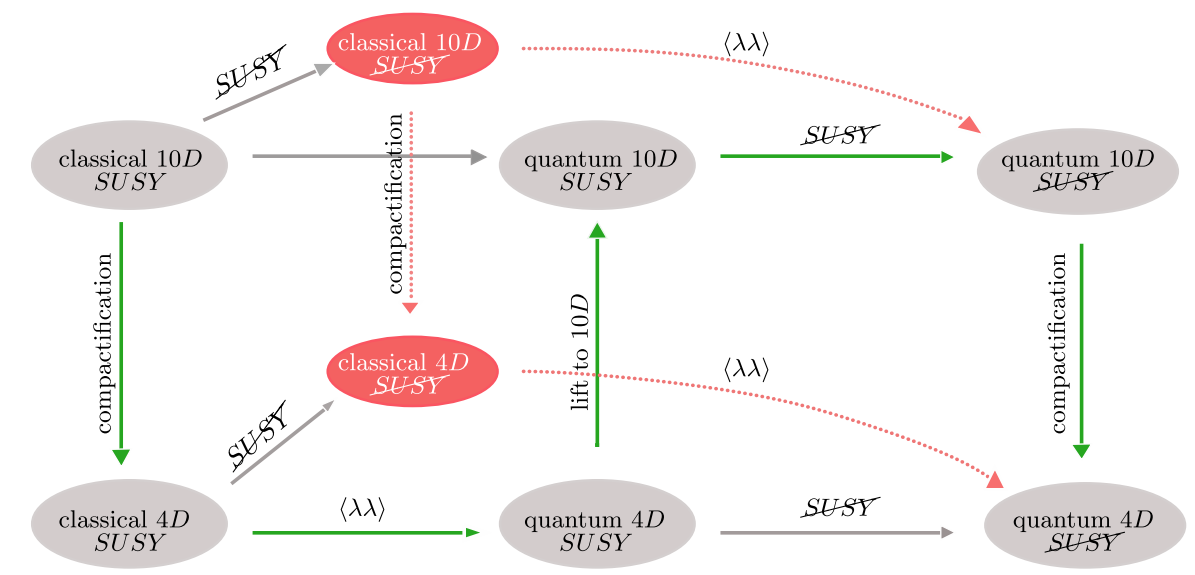

FIG. 2. From the classical 10D supergravity to the quantum 4D theory with broken supersymmetry. Green arrows denote steps that can be followed through unambiguously and with reasonable amount of control. The dimensional reduction of the classical 10D theory is well understood. Incorporating nonperturbative quantum effects in the 4D EFT can be performed with reasonable amount of control as well. The de Sitter uplift of the supersymmetric KKLT vacua within 4D EFT suffers from ambiguities as explained in Sec. II C. For this reason we follow the authors of [65,70-72] in first lifting the SUSY KKLT vacua to 10D. Since at this stage we have a quantum deformed 10D action, we may include the SUSY-breaking effects of an antibrane and thereafter dimensionally reduce the 10D potential to 4D. This prescription avoids the intermediate classical 10D/4D descriptions with a SUSY-breaking runaway potential (marked in red).

position moduli potential (i.e. the backreaction of the D3-brane on the four-cycle size), the 10D approach allows us to also incorporate the backreaction of an $\bar{D} 3$-brane unambiguously (for a diagrammatic representation of the argument, see Fig. 2).

Based on this conclusion, we will investigate whether or not the nonperturbative AdS vacua of KKLT can be uplifted to de Sitter. The approach is to compactify the (quantum corrected) $10 \mathrm{D}$ theory and identify the terms in the corresponding on-shell potential to find a 10D picture of KKLT moduli stabilization, followed by the (partial) uplift.

\section{A. Stabilization of complex structure moduli: ISD solutions}

Flux compactifications of type IIB string theory were pioneered by Giddings, Kachru, and Polchinski [14]. They showed that in the limit of dilute three-form fluxes the Gukov-Vafa-Witten superpotential descends from the 10D potential of type IIB string theory by straightforward dimensional reduction. Moreover, even for nondilute fluxes, the 4D solutions obtained from minimizing the GVW superpotential lift to full 10D solutions, the imaginary self-dual solutions. These feature exponential warping thus realizing the proposal of Randall and Sundrum [77] to naturally generate large hierarchies.

Following their discussion one may derive an on-shell expression for the 4D potential that will prove extremely useful (see appendix A for details). Starting from the 10D Einstein frame action of type IIB supergravity, we make a warped compactification ansatz for the metric

$$
d s^{2}=e^{2 \mathcal{A}(y)} \tilde{g}_{\mu \nu}^{(4)}(x) d x^{\mu} d x^{\nu}+g_{m n}^{6}(y) d y^{m} d y^{n},
$$

and write the self-dual five-form flux as $F_{5}=$ $\left(1+\star_{10}\right) d \alpha(y) \wedge \sqrt{-\tilde{g}^{(4)}} d^{4} x$. The corresponding tracereversed Einstein equations allow us to write the $4 \mathrm{D}$ Ricci scalar $\tilde{R}_{4}$ in terms of the matter content on the compactification. The result can be combined with the Bianchi identity for $F_{5}$ such that

$\tilde{\nabla}^{2} \Phi^{-}=\tilde{R}_{4 D}+\frac{e^{2 \mathcal{A}}}{\operatorname{Im}(\tau)}\left|G_{3}^{-}\right|^{2}+e^{-6 \mathcal{A}}\left|\partial \Phi^{-}\right|^{2}+e^{2 \mathcal{A}} \frac{\Delta^{\mathrm{loc}}}{2 \pi}$,

where

and $\quad \Delta^{\mathrm{loc}} \equiv \frac{1}{4}\left(T_{m}^{m}-T_{\mu}^{\mu}\right)^{\mathrm{loc}}-T_{3} \rho_{3}^{\mathrm{loc}}$.

Here $G_{3}$ is the complexified three-form $F_{3}-\tau H_{3}$. Moreover $T_{M N}^{\text {loc }}$ and $T_{3} \rho_{3}^{\text {loc }}$ are the energy-momentum tensor and $D 3$-brane charge density of localized objects. We work in units such that $\left(M_{\mathrm{P}, 10 D}\right)^{8}=4 \pi$.

By assuming the existence of a solution and demanding its global consistency one obtains an expression for the on-shell potential by integrating the above equation over the internal space

$$
V \cdot M_{\mathrm{P}}^{-4}=\int \frac{d^{6} y \sqrt{g^{6}}}{16 \pi \mathcal{V}_{w} \tilde{\mathcal{V}}_{w}}\left[-e^{8 \mathcal{A}} \frac{\Delta}{2 \pi}-\left|\partial \Phi^{-}\right|^{2}\right],
$$

where 


$$
\begin{aligned}
\Delta & \equiv 2 \pi \frac{\left|G_{3}^{-}\right|^{2}}{\operatorname{Im}(\tau)}+\Delta^{\mathrm{loc}}, \quad \tilde{\mathcal{V}}_{w} \equiv \int_{M_{6}} d^{6} y \sqrt{g^{6}} e^{6 \mathcal{A}}, \quad \text { and } \\
\mathcal{V}_{w} & \equiv \int_{M_{6}} d^{6} y \sqrt{g^{6}} e^{2 \mathcal{A}} .
\end{aligned}
$$

From the on-shell potential (47) it follows immediately that as long as all localized sources satisfy $\Delta^{\text {loc }} \geq 0$ the unique classical Minkowski solutions of type IIB string theory are the ISD solutions,

$$
G_{3}^{-}=\Delta^{\mathrm{loc}}=\tilde{R}_{4 D}=\Phi^{-}=0 .
$$

Under the same assumption de Sitter solutions are ruled out as well. Therefore, a necessary condition for realizing 4D de Sitter solutions is that there exists at least one localized object that satisfies $\Delta^{\text {loc }}<0$.

It is important to note that the ISD solutions also match the 4D no-scale behavior: the volume modulus remains unfixed and corresponds to an overall rescaling of regions of weak warping, leaving strongly warped regions approximately invariant [66]. Therefore, the inclusion of any further sources of positive potential energy cannot lead to a stable solution but must rather lead to decompactification. Therefore, in order to discuss the inclusion of further sources of positive energy, one first needs to incorporate Kähler moduli stabilization.

\section{B. KKLT Kähler moduli stabilization: The 10D perspective}

As explained in the introduction of this section we will determine the effective on-shell potential (47) by dimensionally reducing the 10D action of type IIB supergravity in the presence of a nonvanishing value of the gaugino condensate. In order to do so the following quantities have to be evaluated:

(a) the value of $\Delta^{\text {loc }}$ induced by the nonvanishing gaugino bilinear that appears in the $D 7$-brane action;

(b) the backreaction on the three-form fluxes $G_{3}$ and $\Phi^{-}$. We evaluate these quantities only in the bulk Calabi-Yau where the effects of warping and three-form fluxes are volume suppressed. We will neglect these effects and thus work to leading order in an inverse volume expansion. Moreover we assume the 7-brane configuration to be in the Sen limit [78], i.e. four $D 7$-branes on top of an $O 7$ plane. In this case there is no classical 10D backreaction on the Calabi-Yau geometry and the axiodilaton is constant. The gauge group is $S O(8)$.

The calculation is a somewhat tedious but straightforward exercise that has been partially done by the authors of [70,72]. We have provided the detailed derivation in Appendix $\mathrm{C}$ and merely quote the result here: the piece in the action responsible for the perturbation of the ISD background is ${ }^{6}$

$$
S_{D 7} \supseteq \int_{M_{10}} \pi \delta_{D}^{(0)} e^{\phi / 2} e^{-4 \mathcal{A}} \frac{\bar{\lambda} \bar{\lambda}}{16 \pi^{2}} G_{3} \wedge * \Omega+\text { c.c. }
$$

The action (50) acts as a source for $G_{3}$ and a particular solution to the equations of motion is given by [72]

$$
G_{3}^{\lambda \lambda}=G_{3}^{+}+G_{3}^{-},
$$

with ISD component

$$
e^{4 \mathcal{A}} G_{3}^{+}=\frac{1}{\pi} e^{-\phi / 2} \frac{\langle\lambda \lambda\rangle}{16 \pi^{2}}\left(g^{i \bar{j}} \nabla_{i} \nabla_{\bar{j}} \Psi\right) \bar{\Omega}
$$

and imaginary anti-self-dual (IASD) component

$$
\left(e^{4 \mathcal{A}} G_{3}^{-}\right)_{i \bar{j} \bar{k}}=-\frac{i}{\pi} e^{-\phi / 2} \frac{\langle\lambda \lambda\rangle}{16 \pi^{2}}\left(\nabla_{i} \nabla_{l} \Psi\right) g^{l \bar{m}} \bar{\Omega}_{\bar{m} \bar{j} \bar{k}},
$$

where $\Psi$ is determined by $\nabla^{2} \Psi=2 \pi \delta(\Sigma)$, and $\delta(\Sigma)$ is the scalar delta function that localizes on the four-cycle that the 7-branes wrap. ${ }^{7}$ Note that $\Psi$ is identified with $\operatorname{Re} \log h\left(z^{i}\right)$, where $h\left(z^{i}\right)=0$ is the holomorphic embedding equation of the 7-brane divisor [70]. The flux profile (51) is only a particular solution to the equations of motion and is completed by the global harmonic fluxes.

Building on these results we now proceed. In order to match the 4D description of KKLT we should be able to determine the value of the volume modulus at the supersymmetric minimum from the value of the GVW superpotential. In order to do this in general one would have to derive the full off-shell potential (26) while we derive only the on-shell potential (47). Fortunately if we assume a relation between the condensate and the 4-cycle volume $\langle\lambda \lambda\rangle \sim e^{i a \rho}$ we may deduce the value of $\rho$ by demanding that the quantum deformed 10D SUSY conditions are fulfilled. These were derived in [72] and we merely quote their result: to leading order in the gaugino condensate, the three-form flux $G_{3}$ is given by

\footnotetext{
${ }^{6}$ Note that we extrapolate the Abelian $D 7$-brane term to the non-Abelian case. $\lambda \lambda$ will be shorthand for $\operatorname{Tr}\left(\lambda^{\alpha} \lambda_{\alpha}\right)$. We would like to stress that the most powerful argument for the validity of this approach comes not from the fact that this term can be obtained from the (non-Abelian) 7-brane action but rather from the $10 \mathrm{D} \leftrightarrow 4 \mathrm{D}$ matching of the $D 3$-brane position moduli potential. Therefore the extrapolation of the non-Abelian 7-brane action is no further reason of concern for us here.

${ }^{7}$ Strictly speaking, $\Psi$ is the solution to $\nabla^{2} \Psi=$ $2 \pi\left(\delta(\Sigma)-\frac{\operatorname{Vol}(\Sigma)}{\mathcal{V}}\right)[65]$. We will be interested in the behavior of supergravity fields in the vicinity of localized objects. In this regime the constant correction to the source of $\Psi$ only gives rise to small (i.e. volume suppressed) corrections which we shall neglect consistently.
} 


$$
G_{3}=G_{3}^{(2,1)}+G_{3}^{\lambda \lambda}
$$

where $G_{3}^{(2,1)}$ is harmonic and of Hodge type $(2,1)$. Thus, the $(0,3)$ component of $G_{3}$ localizes completely on the 7-brane divisor and is related to the value of the gaugino condensate via the $G_{3}$ equations of motion. As a consequence, just as in the 4D EFT description the value of the Gukov-Vafa-Witten superpotential determines the value of the condensate,

$$
W_{0} \sim \int_{M_{6}} G_{3} \wedge \Omega \sim e^{-\phi / 2}\langle\lambda \lambda\rangle \sim e^{i a \rho}
$$

Apart from the harmonic $(2,1)$ component the $G_{3}$ flux profile is thus fully determined. Furthermore one may show that the field $\Phi^{-}$is not perturbed to leading order in the condensate.

$\Delta^{\mathrm{loc}}$ is given by

$$
\Delta_{\mathrm{loc}}=-\frac{3 \pi}{4} e^{\phi / 2} e^{-4 \mathcal{A}} \frac{\langle\bar{\lambda} \bar{\lambda}\rangle}{16 \pi^{2}} \Omega \cdot G_{3} \delta_{D}^{(0)}+\text { c.c. }
$$

This means that all the ingredients of the on-shell potential are gathered and after some algebra (see Appendix C) we arrive at

$$
V \cdot M_{\mathrm{P}}^{-4}=-\int_{M_{6}} \frac{d^{6} y \sqrt{g}}{8 \pi^{3} \mathcal{V}_{w} \tilde{\mathcal{V}}_{w}}\left|\frac{\langle\lambda \lambda\rangle}{16 \pi^{2}} \nabla_{i} \nabla_{j} \Psi\right|^{2}
$$

Just as we know from the 4D EFT analysis the cosmological constant is negative and proportional to the strength of the gaugino condensate.

One can readily generalize this expression to the case of $n$ stacks of 7-branes that wrap different holomorphic representatives of the same divisor,

$$
\begin{aligned}
V \cdot M_{\mathrm{P}}^{-4}= & -\int \frac{d^{6} y \sqrt{g}}{8 \pi^{3} \mathcal{V}_{w} \tilde{\mathcal{V}}_{w}}\left(4\left|\sum_{a=1}^{n} \frac{\langle\lambda \lambda\rangle_{a}}{16 \pi^{2}} \nabla_{i} \nabla_{j} \Psi_{a}\right|^{2}\right. \\
& \left.-3 \sum_{a=1}^{n}\left|\frac{\langle\lambda \lambda\rangle_{a}}{16 \pi^{2}} \nabla_{i} \nabla_{j} \Psi_{a}\right|^{2}\right) .
\end{aligned}
$$

Crucially, the sign of the cosmological constant depends on the relative phases between the condensates $\langle\lambda \lambda\rangle_{a}$. This suggests that for two condensates one may be able to obtain a cosmological constant that is parametrically smaller than the strength of the individual condensates that set the mass scale of the lightest modulus. For more than two condensates it may even be possible to obtain de Sitter solutions.

This fits nicely with the effective 4D description of multiple condensates: the case of two condensates was studied by Kallosh and Linde [37] who show that it is possible to tune the cosmological constant to zero supersymmetrically while retaining a finite volume stabilization.

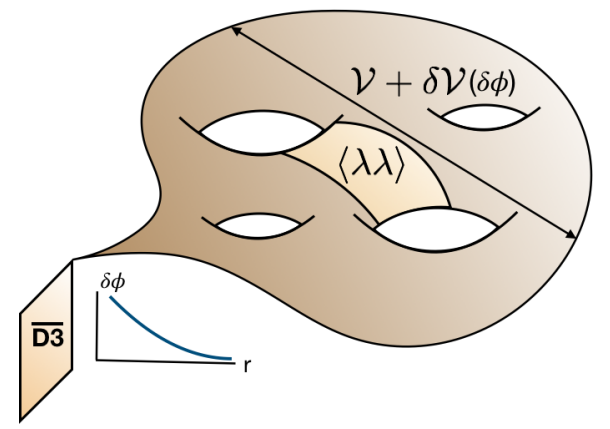

FIG. 3. Two aspects of $\overline{D 3}$ backreaction: on the one hand the local throat perturbations sourced by the $\overline{D 3}$-brane at the bottom of the throat fall off exponentially towards the bulk Calabi-Yau. Their UV tail can be neglected in our analysis. On the other hand the overall volume of the bulk Calabi-Yau adjusts and so does the condensate $\langle\lambda \lambda\rangle$. This global backreaction effect turns out to be crucial for our analysis.

Before we proceed one more comment is in order: the integrals (57) and (58) are quadratically UV divergent. Imposing a UV-cutoff $\Lambda_{\mathrm{UV}}$ one finds

$$
|V| \cdot M_{\mathrm{P}}^{-4} \sim \frac{\operatorname{Vol}\left(\Sigma_{4}\right)}{\mathcal{V}^{2}}\left|\frac{\langle\lambda \lambda\rangle}{16 \pi^{2}}\right|^{2} \Lambda_{\mathrm{UV}}^{2}+\text { finite. }
$$

Since we are not interested in overall order one coefficients we leave a proper EFT treatment of this divergence for future work and simply cut off the integrals at the string scale $^{8} \Lambda_{\mathrm{UV}}^{2} \sim e^{\phi / 2}$. We note that this divergence is analogous to the divergent self-energy of an antibrane at the bottom of a warped throat which is explained in [34,35].

\section{The uplift}

In order to determine if an antibrane (or in fact any other source of SUSY breaking) at the bottom of a warped throat can uplift to de Sitter in the presence of only a single condensate, we have to take a closer look at the on-shell potential (47). The antibrane perturbs it in two ways (see Fig. 3).

(a) It sources all local 10D supergravity fields in the throat. Away from the brane their field profiles are harmonic and fall off towards the bulk CY.

(b) It pulls on the volume modulus, the lightest degree of freedom in the problem. Because we assume an exponential relation between the condensate and the volume modulus, the value of the condensate is changed as well.

Let us focus on the local backreaction first. The naive approach to include the effect of the $\overline{D 3}$-brane would be to compute its corresponding contribution to the on-shell potential. The outcome is $\Delta \frac{\text { loc }}{D 3}=2 T_{3}>0\left(T_{3}\right.$ is the tension

\footnotetext{
${ }^{8}$ Recall that we work in $10 \mathrm{D}$ Einstein frame in units $M_{\mathrm{P}, 10 D}^{8}=4 \pi$.
} 
of the brane), so the inclusion of the $\overline{D 3}$-brane implies a new negative contribution to (47). Then, how is the antibrane going to provide an uplift? As we already saw in the toy model, the use of on-shell potentials comes with certain peculiarities: The dominant source of uplift (the three-brane tension) did not appear explicitly in the on-shell potential. Rather its uplifting effect is contained implicitly in the induced shift in the volume modulus by which the uplift can be efficiently parametrized even when the backreacted solution is not known. Now, we are in a similar situation. The explicit appearance of the antibrane via $\Delta^{\text {loc }}$ and in fact all the details of the backreacted warped throat geometry are suppressed by eight powers of the warp factor. ${ }^{9}$ We know from the off-shell potential (26) that the leading order uplift should be of order $e^{4 \mathcal{A}_{0}} T_{3}$, and therefore, if we only work to order $e^{4 \mathcal{A}_{0}}$ we can completely neglect the local physics of the throat, making the sign of $\Delta \frac{\text { loc }}{D 3}$ irrelevant. Instead, as in the toy example, the leading order uplift is parametrized implicitly by the induced backreaction on the bulk Calabi-Yau on which we will focus in the following.

Before doing so let us set a precision goal for the upcoming analysis. Because the minimal uplifting potential must compete with the AdS depth of the SUSY KKLT minimum one should have $e^{4 \mathcal{A}_{0}} \gtrsim|\langle\lambda \lambda\rangle|^{2}$ as a minimal requirement. Guided by this in the following we will work to leading order in

$$
e^{(4-2 q) \mathcal{A}_{0}}|\langle\lambda \lambda\rangle|^{q},
$$

for any $q$ and consistently neglect higher combined powers of the condensate and the warp factor.

The next step is to estimate how the local physics of the bulk is affected by the SUSY-breaking source in the strongly warped region. Of course one cannot use on-shell methods to do this. But since the supergravity solution corresponding to a warped throat is known in full detail, this estimate can be done explicitly by perturbing the IR end of the throat and deriving how fast the corresponding field profiles fall off towards the UV. Building on the work of $[70,79,80]$ we have provided a treatment of this question in Appendix E.

The outcome is simple and intuitive: all field profiles fall off exponentially towards the bulk such that for an IR perturbation of order one the corresponding perturbation in the UV is suppressed by powers of the IR warp factor $e^{\mathcal{A}_{0}}$. If we neglect dependencies on the $\mathrm{CY}$ volume which we assume to be only moderately large it, turns out that to leading order in $e^{\mathcal{A}_{0}}$ all local supergravity fields receive corrections of order

\footnotetext{
${ }^{9}$ Note that close to the anti- $D 3$-brane the potential $\Phi^{-}$is sourced at $\mathcal{O}\left(e^{4 \mathcal{A}_{0}}\right)$. Hence, the integrand of the on-shell potential is dressed with an overall factor of $e^{8 \mathcal{A}}$.
}

$$
\mathcal{O}\left(e^{p \mathcal{A}_{0}} \cdot T_{3}\right), \quad p \in\{3,4\} .
$$

Because both $G_{3}^{-}$as well as $\Phi^{-}$appear quadratically in (47), we may neglect their adjustment that is sourced directly by the antibrane. Also the localized contribution in (56) contains an overall factor of $\langle\lambda \lambda\rangle$. Hence, local field profiles would have to be corrected at least at order $e^{2 \mathcal{A}_{0}}$ to enter our discussion. No such field profiles can be sourced directly by the antibrane.

Hence, we need only consider the adjustment of the universal Kähler modulus. However, calculating the value of the volume modulus is not possible using our on-shell methods and we lack the required off-shell methods to explicitly determine backreaction effects in the bulk. The only reason that we were able to deduce its value at the supersymmetric point and relate it to the value of the GVW superpotential was that the 10D SUSY conditions gave us enough constraints. At no point did we explicitly minimize an effective off-shell potential of the volume modulus.

Let us argue why we are able to proceed despite this shortcoming of the on-shell methods. First, the validity of the on-shell methods as such does not require supersymmetry. We assume that once the antibrane is included all fields adjust to their new respective minima. Hence once the system has found its energetically most favorable configuration, the outcome must be consistent with the on-shell potential (47). The only technical difference between the supersymmetric and nonsupersymmetric configuration is that we are no longer able to determine the shifted value of the volume modulus. For our purposes this is not a problem. We simply assume that the new minimum lies at a different volume

$$
\rho \rightarrow \rho+\delta \rho,
$$

and express all the quantities that need to be evaluated as functions of the new (unknown) value of the volume modulus. When the volume modulus assumes a new value, all 7-brane sourced effects adjust accordingly. Discarding effects that are subleading in inverse volume (to be addressed shortly), this means that in configurations with one or more gaugino condensates stabilizing the Kähler modulus, upon the inclusion of the antibrane, equations (57) and (58) still hold albeit as a function of the (unknown) shifted value of the volume modulus. Hence, from (57) it is easy to see that in the case of a single gaugino condensate even in the presence of the antibrane the on-shell potential is manifestly negative.

Ultimately, the reason that the contribution of a single gaugino condensate to $V$ is always negative rests on the fact that its contribution to the integrand of the on-shell potential is always negative ${ }^{10}$ (see Appendix $\mathrm{C}$ for details). Although

\footnotetext{
${ }^{10}$ The reason for this is that the 7-brane contribution to $\Delta \equiv 2 \pi \frac{\left|G_{3}^{-}\right|^{2}}{\operatorname{Im}(\tau)}+\Delta^{\mathrm{loc}}$ is positive.
} 
there are two 7-brane-induced contributions to the integrand of either sign from the induced bulk and brane-localized fluxes, the negative contribution of the 7-brane-induced bulk piece $G_{3}^{-}$overcompensates the 7-brane-localized $G_{3}^{+}$piece. This happens for all values of $\rho$ as long as both $G_{3}^{+}$and $G_{3}^{-}$are sourced by a unique 7-brane world volume coupling of the condensate $\langle\lambda \lambda\rangle$ to $G_{3}$. We have shown in Appendix $C$ that in absence of any other fermion condensates besides $\langle\lambda \lambda\rangle$, the coupling $\langle\lambda \lambda\rangle G_{3} \cdot \Omega$ is unique at $\mathcal{O}(\langle\lambda \lambda\rangle) .{ }^{11}$ This statement holds at the level of the 10D equations of motion and does not use background supersymmetry for the derivation.

Hence, in the end the situation here is analogous to the Freund-Rubin toy model (without the 5-branes) and also here it follows immediately that vacua that are stabilized by a single condensing non-Abelian gauge group (in a regime where perturbative corrections can be neglected, see Sec. V) cannot be uplifted to de Sitter.

As we have explained we cannot in principle derive the adjusted value of the volume modulus and the corresponding change in the cosmological constant from the on-shell potential. The on-shell potential (57) only reveals the overall sign of the resulting cosmological constant but does not allow one to compute its value. For this one would need the full off-shell potential which we currently lack. Nevertheless, we can attempt a parametric estimate (neglecting volume powers) based on generic assumptions about the coupling of an uplift parametrized by the warped tension $e^{4 \mathcal{A}_{0}} T_{3}$ to the volume modulus $\rho$ (see Appendix E)

$$
\delta \rho \sim \frac{e^{4 \mathcal{A}_{0}} T_{3}}{m_{\rho}^{2}} \sim \frac{e^{4 \mathcal{A}_{0}} \cdot T_{3}}{|\langle\lambda \lambda\rangle|^{2}} .
$$

This estimate is easy to understand: the weaker the stabilization the stronger the volume modulus will react to a small IR perturbation. Therefore for $e^{4 \mathcal{A}_{0}} T_{3} \ll|\langle\lambda \lambda\rangle|^{2}$ we can plug this estimate into the on-shell potential (57) to obtain

$$
\delta V \sim \underbrace{V}_{\sim|\langle\lambda \lambda\rangle|^{2}} \cdot \underbrace{\delta \rho}_{\sim e^{4 \mathcal{A}_{0} \cdot T_{3} /|\langle\lambda \lambda\rangle|^{2}}} \sim e^{4 \mathcal{A}_{0}} \cdot T_{3}
$$

which describes the naive result of adding energy contributions. Note that this regime corresponds precisely to the region of parametric control where the uplift is small compared to the initial vacuum energy $\delta V \ll\left|V_{\text {AdS }}\right|$ and thus the final state is also AdS.

When the warped tension is not much smaller then $\left|V_{\text {AdS }}\right|$ it is not possible even to estimate the magnitude of the uplift since $\delta \rho$ is no longer small. From the on-shell potential we only know that the final state will be 4D AdS and the inclusion of the antibrane should increase the

\footnotetext{
${ }^{11}$ We use the straightforward non-Abelian generalization of the single $D 7$-brane outcome, guided by our expectation that gauge symmetry and supersymmetry will uniquely fix this.
}

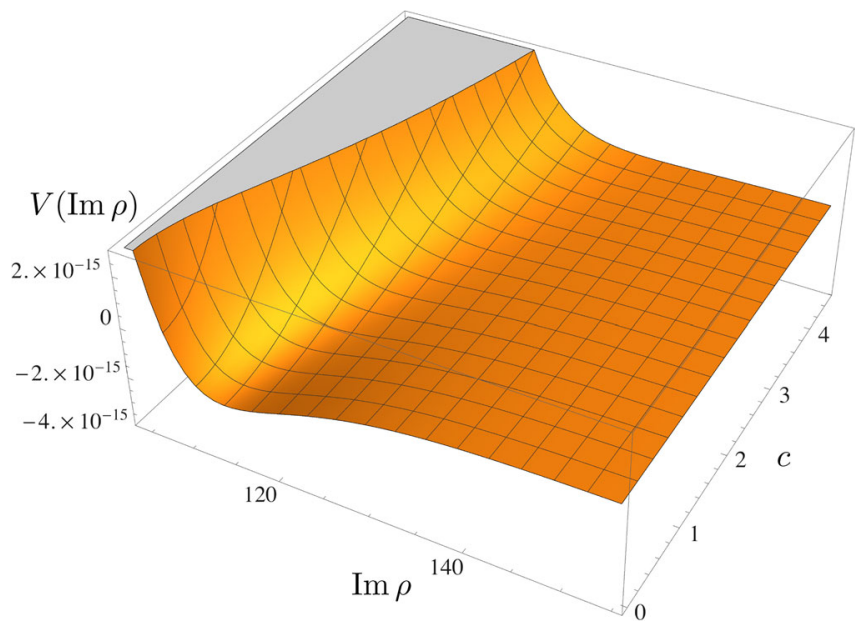

FIG. 4. The $F$-term potential of (17) for the case $b=0$ as a function of the uplift parameter $c$. In this case the superpotential reads $W=W_{0}+A(1+c S) \exp (i a \rho)$. The $\rho$ minimum moves out to larger values and stays $\mathrm{AdS}$ as $c$ increases. This behavior is compatible with our 10D analysis but does not uniquely follow from it.

potential energy. From here we conclude that its magnitude will be flattened out due to strong backreaction on the volume modulus and thus the final energy is not just the sum of $V_{\text {AdS }}$ and the warped antibrane tension.

It is now interesting to go back to the $4 \mathrm{D}$ superpotential (17) to note that our $10 \mathrm{D}$ analysis is compatible with the extreme case $b \rightarrow 0$, suggesting the existence and significant strength of the superpotential term describing the interaction between the antibrane and the gaugino condensate (see Fig. 4). We will comment further on this in Sec. IV D.

For completeness, we would like to comment here on an interesting effect of the volume shift caused by the $\overline{D 3}$-brane. We are assuming an exponential relation between the condensate $\langle\lambda \lambda\rangle$ and the volume modulus, and so the condensate must be reduced in magnitude due to the shift. Since the profile $G_{3}^{\lambda \lambda}$ is uniquely determined by the strength of the gaugino condensate, the situation is so far analogous to the supersymmetric case except that we have to allow for a global harmonic $(0,3)$ component in the three-form

$$
G_{3}=G_{3}^{(2,1)}+G_{3}^{(0,3)}+G_{3}^{(\lambda \lambda)} .
$$

The global $(0,3)$ component enters the on-shell potential (47) via $\Delta^{\text {loc }}$ and thus gives rise to a further term in the onshell potential. The magnitude of the global $(0,3)$ component is determined as a function of the shifted volume by demanding the value of $\int G_{3} \wedge \Omega$ to be conserved. ${ }^{12}$ Since the localized $(0,3)$ piece is reduced in comparison

\footnotetext{
${ }^{12}$ Here we assume that backreaction on complex structure moduli is negligible.
} 
with the supersymmetric setup, part of it is converted into the global $(0,3)$ component. This harmonic $(0,3)$ component is interesting because it determines soft masses on branes [81-83] of order the warped tension of the antibrane. For our purposes, it enters the on-shell potential (47) via $\Delta^{\text {loc }}$ only with a relative volume suppression with respect to e.g. the localized flux contribution on the D7-brane stack (see Appendix D for details). We work to leading order in $1 / \mathcal{V}$ and therefore neglect this contribution.

The obstruction towards reaching de Sitter in the single condensate configuration can be evaded in a rather simple generalization of the setup: in racetrack configurations with at least two condensing non-Abelian gauge groups the multicondensate potential (58) confirms from a $10 \mathrm{D}$ perspective the possibility to decouple the lightest modulus mass from the AdS depth in the supersymmetric vacuum by giving the individual condensates opposite phases. In a limiting case it may even allow for Minkowski vacua after stabilizing the Kähler modulus. This situation would correspond to the case when the two terms in (58) are of the same magnitude upon integration and thus compensate each other. Note that in this case there exists the required positive contribution to the on-shell potential, allowing one to evade the Maldacena-Núñez theorem, in analogy with the Freund-Rubin toy model when the 5-brane is present. Therefore, the inclusion of the $\overline{D 3}$-brane on the bottom of a warped throat in this configuration could well provide the necessary uplift to de Sitter (see Fig. 5).

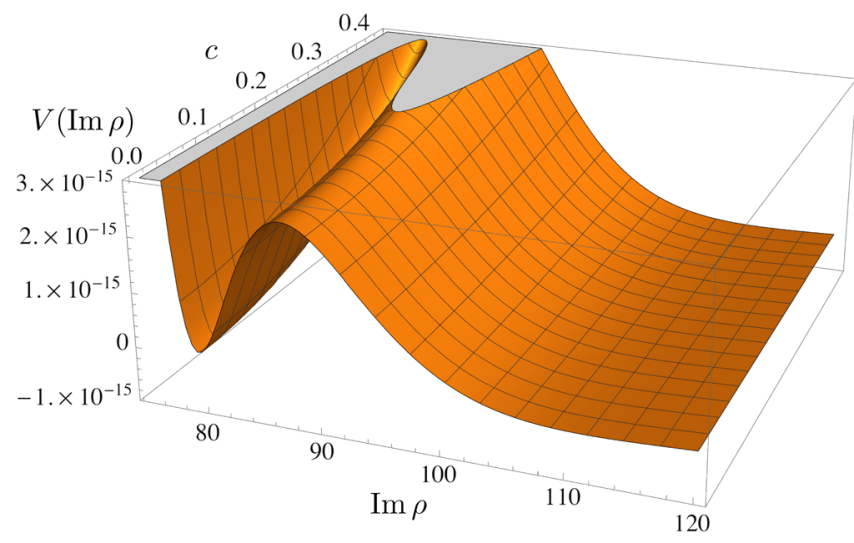

FIG. 5. The $F$-term potential analogous to (17) for the case $b=0$ as a function of the uplift parameter $c$ for racetrack superpotential. In this case the superpotential reads $W=$ $W_{0}+A_{1}(1+c S) \exp \left(i a_{1} \rho\right)+A_{2}(1+c S) \exp \left(i a_{2} \rho\right)$. For simplicity we have put the coefficients of the $S$ dependence in both gaugino condensates equal. For vanishing uplift $c=0$ the scalar potential shows the tuned racetrack Minkowski minimum at smaller values of $\rho$ then the coexisting KKLT AdS minimum at larger values or $\rho$. Clearly, the racetrack minimum gets successfully uplifted to de Sitter even for $c \ll 1$ while the KKLT minimum at larger $\rho$ continues to move out to larger values and stays AdS as $c$ increases. Note that this is a 4D effective extrapolation to the two-condensate case, for which we do not have a fully matching 10D description yet.
While we cannot prove the full 10D consistency of such racetrack stabilization mechanisms we see no sign of failure in an anticipated fully fledged backreacted 10D solution. If the required tuning can be realized it would seem that such vacua can be uplifted to de Sitter generically (as explained in Sec. II C), i.e. by any SUSY-breaking energy density that is redshifted sufficiently strongly to suppress backreaction on the volume modulus (i.e. $e^{4 \mathcal{A}_{0}} \ll\left|\langle\lambda \lambda\rangle_{a}\right|^{2}$ ). Thus, it seems that such two-condensate (racetrack) stabilization mechanisms are ideal backgrounds for uplifting scenarios, independently of the details of the uplifting mechanism, and the details of its couplings to the lightest moduli. However, we would also like to point out that the mere existence of warped throats with warping in the regime $e^{4 \mathcal{A}_{0}} \ll\left|\langle\lambda \lambda\rangle_{a}\right|^{2}$ is not obvious.

\section{Towards an interpretation of an unsuppressed $S\langle\lambda \lambda\rangle$ coupling}

We would like to conclude this section with an attempt of a physical interpretation of our result. We have constrained the anticipated fully fledged backreacted solution using on-shell methods. Although these methods are powerful enough to constrain the sign of the cosmological constant, they reveal very little about its physical origin. So, let us speculate about it:

We are assuming that near the bottom of the warped throat the local description of a warped throat à la Klebanov-Strassler still holds, while at the same time we believe that the 4D EFT description of the antibrane state using a nilpotent superfield is valid. Putting these two expectations together, and recalling the superpotential of Eq. (18), we are led to the interpretation that the IR warp factor itself receives corrections of order $|\langle\lambda \lambda\rangle|$, i.e.

$$
e^{2 \mathcal{A}_{0}} \rightarrow e^{2 \mathcal{A}_{0}}+\mathcal{O}(|\langle\lambda \lambda\rangle|)
$$

We have asked ourselves if this expectation is reasonable in view of considerable effort that has been put into determining the position moduli potential of D3-branes [70] and $\overline{D 3}$-branes [80]. In principle, the quantum corrected warp factor can be reconstructed by adding the $D 3$ and $\overline{D 3}$ potentials. In [70] it was found that the D3-brane moduli potential as induced by the nonperturbative bulk effects is of order $e^{\mathcal{A}_{c l, 0}}|\langle\lambda \lambda\rangle|^{2}$ and is hence suppressed by a classical warp factor. However, to the best of our knowledge, the tools used in [70] are only sensitive to the force that acts on the D3-brane, that is only the position-dependent part of the $D 3$-brane moduli potential is determined. Their results are hence compatible with the correction that we have suggested.

If this correction indeed occurred, an interesting consequence would be that the IR warp factor receives significant corrections in the regime $e^{4 \mathcal{A}_{0}} \sim|\langle\lambda \lambda\rangle|^{2}$. Nevertheless, the local description of the IR region of the warped throat 
would stay intact as long as the weaker requirement $|\langle\lambda \lambda\rangle|^{2} \ll e^{3 \mathcal{A}_{0}}$ is fulfilled. ${ }^{13}$ A local throat observer would notice the effect we are suggesting merely as a change of Newton's constant.

An interesting prospect for future research would be to validate or falsify our interpretation by finding the explicit backreacted warped throat solution. The tools needed to do this have been laid out in [79] who have put the type IIB supergravity equations of motion into "triangular" form suitable for determining the response of the throat to a source term in a systematic fashion. Following their strategy one could expand the fields $\Phi^{-}$and $\Phi^{+}$that determine the backreacted warp factor as

$$
\begin{gathered}
\Phi^{-}=\underbrace{\left.\Phi^{-}\right|_{I S D}}_{=0}+\left.\delta \Phi^{-}\right|_{\mathcal{I H}}+\left.\delta \Phi^{-}\right|_{\mathcal{H}}, \\
\left(\Phi^{+}\right)^{-1}=\underbrace{\left.\left(\Phi^{+}\right)^{-1}\right|_{I S D}}_{=\left.\frac{1}{2} e^{-4 A}\right|_{I S D}}+\left.\delta\left(\Phi^{+}\right)^{-1}\right|_{\mathcal{I H}}+\left.\delta\left(\Phi^{+}\right)^{-1}\right|_{\mathcal{H}},
\end{gathered}
$$

where the subscripts $\mathcal{I H}$ and $\mathcal{H}$ denote the inhomogeneous, respectively homogeneous, part. Integrating the equations of motion would determine $\Phi^{-}$as well as $\Phi^{+}$from which the warp factor can be determined uniquely.

\section{APPLYING THE LESSONS: DE SITTER IN KKLT WITH $\alpha^{\prime}$ CORRECTIONS?}

Let us use the lessons learned from the previous analysis to speculate about alternatives to the racetrack setup in the language of 4D EFT. From the type IIB analysis in [84,85] as well as more recent lifts to $F$-theory in [86], we know that the $R^{4}$ terms and their supersymmetric completion in 10D induce the leading correction to the 2-derivative kinetic terms of the Kähler moduli at $\mathcal{O}\left(\alpha^{\prime 3}\right)$.

The results of [87] demonstrated that other $\alpha^{\prime}$ corrections involving higher powers of the Ramond-Ramond (RR) and/or Neveu-Schwarz-Neveu-Schwarz (NSNS) $p$-form field strengths produced 4D contributions suppressed by additional inverse powers of the compactification volume, while the results of [88-93] show that the same is true for string loop corrections due to the extended no-scale structure present in type IIB Calabi-Yau compactifications. As a result, the Kähler potential of the volume moduli acquires at leading order an $\mathcal{O}\left(\alpha^{\prime 3}\right)$ correction

$K=-2 \ln (\mathcal{V}+\xi / 2)+K_{\text {c.s. }} \quad$ with $: \quad \xi \sim-g_{s}^{-3 / 2} \chi_{\mathrm{CY}}$

where $\chi_{\mathrm{CY}}=2\left(h^{11}-h^{21}\right)$ is the Euler characteristic of the Calabi-Yau in question.

We again assume supersymmetric flux stabilization of the c.s. moduli and axiodilaton. We then proceed to use the

\footnotetext{
${ }^{13}$ If this requirement is violated, local IASD field strengths would become dominant [70].
}

superpotential of KKLT $W=W_{0}+A e^{i a \rho}$. The resulting scalar potential is (see $[38,84]$ )

$$
\begin{aligned}
V_{F}= & e^{K}\left[K^{\rho \bar{\rho}}\left(a^{2} A^{2} e^{i a(\rho-\bar{\rho})}+i a A\left(e^{i a \rho} \bar{W} K_{\bar{\rho}}-e^{-i a \bar{\rho}} W K_{\rho}\right)\right)\right. \\
& \left.+3 \xi \frac{\xi^{2}+7 \xi \mathcal{V}+\mathcal{V}^{2}}{(\mathcal{V}-\xi)(2 \mathcal{V}+\xi)^{2}}|W|^{2}\right] \\
= & \frac{e^{K_{\text {c.s. }} .}}{8 \sigma^{3}}\left[\frac{4 \sigma^{2}}{3}\left(a^{2} A^{2} e^{-2 a \sigma}+2 a A e^{-a \sigma} \operatorname{Im}\left(e^{-i a \operatorname{Re} \rho} W K_{\rho}\right)\right)\right. \\
& \left.+3 \xi \frac{\xi^{2}+7 \xi \sigma^{3 / 2}+\sigma^{3}}{\left(\sigma^{3 / 2}-\xi\right)\left(2 \sigma^{3 / 2}+\xi\right)^{2}}|W|^{2}\right]
\end{aligned}
$$

where we defined $\sigma \equiv \operatorname{Im} \rho=\frac{1}{2} \mathcal{V}^{2 / 3}$. We plot this potential as a function of $\sigma$ for the parameter choice of the original numerical example in KKLT: $a=0.1, A=1, W_{0}=-10^{-4}$ in Fig. 6. We see that the $\alpha^{\prime}$ correction only starts to modify the vacuum energy of the SUSY KKLT vacuum significantly once $\xi$ is large enough to violate $\xi \ll \mathcal{V}$.

Looking at the figure, we observe the following. First, $m_{\rho}$ is determined roughly by the whole potential difference between the minimum and the barrier top. Second, by arranging for $\xi \rightarrow \mathcal{V}$ we can get a finite hierarchy between the AdS vacuum energy of the minimum and the total potential difference between the minimum and the barrier top $\left|V_{\text {AdS }}\right| \gtrsim 1 / 3 \Delta V_{\text {tot }}<\Delta V_{\text {tot }}$ where $\Delta V_{\text {tot }}=V_{\text {barrier }}-V_{\text {top }}$. Putting this together, this implies that we can arrange for a finite hierarchy of the necessary dS uplift $\delta V \sim\left|V_{\text {AdS }}\right| \sim$ $1 / 3 \Delta V_{\text {tot }}<\Delta V_{\text {tot }} \sim m_{\rho}^{2} M_{\mathrm{P}}^{2}$.

As this hierarchy was the condition for perturbatively small uplifting to de Sitter which we learned from the

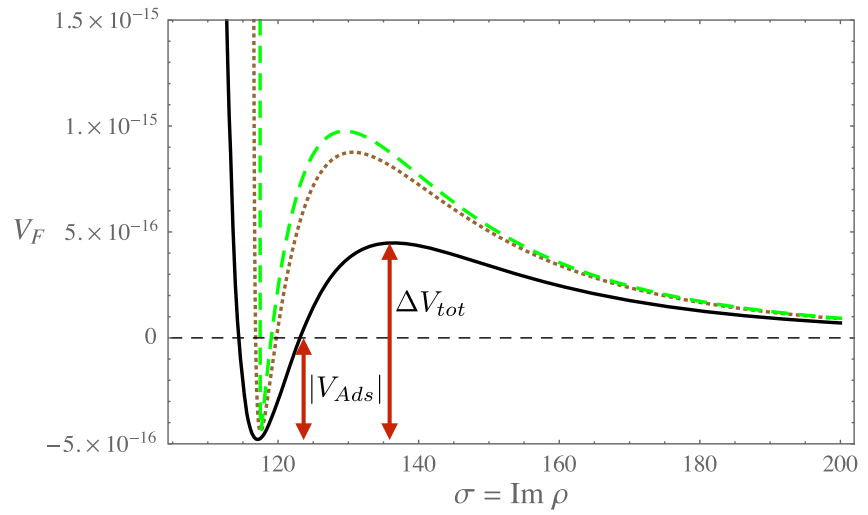

FIG. 6. Volume stabilization in KKLT with a single gaugino condensate but including the leading $\mathcal{O}\left(\alpha^{\prime 3}\right)$ correction to the volume moduli effective action. For $\xi \rightarrow \mathcal{V}$ we see that the correction begins to create a barrier towards decompactification at positive values of the potential. We see that the total potential difference between the SUSY minimum and the barrier top can become as large as about $4 \times\left|V_{\text {AdS }}\right|$. The curves show the potential for values of $\xi=3.1 \times 10^{3}$ (black solid line), $\xi=3.55 \times 10^{3}$ (brown dotted line), and $\xi=3.6 \times 10^{3}$ (green dashed line). 
preceding discussion, it is possible that this simple modification of KKLT with a single gaugino condensate for each volume modulus might allow for perturbatively controlled uplifts to de Sitter. Note, however, that for the hierarchy $m_{\rho}^{2} M_{\mathrm{P}}^{2}>\left|V_{\text {AdS }}\right|$ to emerge, we need to have $\xi \simeq \mathcal{V}$. This is a regime where we might worry about further, higher $\alpha^{\prime}$ corrections no longer being smaller than the leading one, so further analysis is necessary to clarify the viability of this modification of KKLT to get to de Sitter.

Given these observations, it is tempting to speculate about the situation in models where the $\alpha^{\prime}$ correction to $K$ is under parametrical control, such as the large volume scenario (LVS) [38]. In its simplest version it involves a CY with two Kähler moduli $\rho_{b}$ and $\rho_{s}$ and a volume of swiss cheese form

$$
\mathcal{V}=\sigma_{b}^{3 / 2}-\sigma_{s}^{3 / 2}
$$

where we denote the four-cycle volumes as $\sigma_{i}=\operatorname{Im} \rho_{i}$. Furthermore, the only relevant nonperturbative contribution to $W$ will wrap $\sigma_{s}$

$$
W=W_{0}+A_{s} e^{i a_{s} \rho_{s}} .
$$

For this setup there exists for generic values of $\left|W_{0}\right| \sim \mathcal{O}(1)$ a minimum for both $\mathcal{V}$ and $\sigma_{s} \sim \xi^{2 / 3} \sim \mathcal{O}(10)$ at exponentially large volume

$$
\mathcal{V} \sim\left|W_{0}\right| e^{a_{s} \sigma_{s}} \gg \sigma_{s}^{3 / 2}
$$

The interesting observation for our purpose here is that this minimum is a SUSY-breaking AdS minimum with the following scales

$$
\left|V_{\mathrm{AdS}}\right| \sim \frac{\left|W_{0}\right|^{2}}{\mathcal{V}^{3}} \sim m_{\mathcal{V}}^{2} M_{\mathrm{P}}^{2} \sim m_{\sigma_{b}}^{2} M_{\mathrm{P}}^{2} \ll m_{\sigma_{s}}^{2} M_{\mathrm{P}}^{2} \sim \frac{\left|W_{0}\right|^{2}}{\mathcal{V}^{2}} .
$$

Hence, in the LVS setup the amount of antibrane uplift $\delta V \sim\left|V_{\mathrm{AdS}}\right| \sim \mathcal{V}^{-3} \ll m_{\sigma_{s}}^{2} M_{\mathrm{P}}^{2}$ is much smaller than the mass scale of the only four-cycle volume directly controlling the scale of a nonperturbative effect.

Naively, this seems to satisfy the upshot of our preceding 4D and 10D analysis, namely the controlled-uplift condition $\delta V \ll m_{\phi_{i}}^{2} M_{\mathrm{P}}^{2}$ for all moduli $\phi_{i}$ involved in nonperturbative moduli stabilization dynamics. However, LVS setups involve always at least two volume moduli, and their mechanism uses the $\alpha^{\prime}$ correction to produce one volume modulus with exponentially enhanced vacuum expectation value (VEV) via an exponential lever arm in terms of a small but heavy blow-up volume modulus. At this point it is therefore far from clear that the naive scale separation between the necessary amount of uplift in LVS models and the parametrically heavier blow-up four-cycle controlling the leading nonperturbative effect will shield the light overall volume direction sufficiently from the backreaction effects of uplift to conclude a higher level of safety of LVS compared to KKLT models with a single nonperturbative effect per four-cycle.

Another important point pertains to the fact that the inclusion of the $\alpha^{\prime}$ correction is so far only possible in 4D EFT, given that its effect on the scalar potential only emerges after compactification. It is an open question how to include such $\alpha^{\prime}$ corrections from higher-curvature terms at the level of the 10D description directly. Hence, we leave the analysis of the 10D stability of scenarios like LVS under uplifting as a tantalizing problem for the future.

\section{CONCLUSION}

In this paper we have reported progress towards a 10D description of the steps involving volume moduli stabilization without and with anti-D3-brane uplifting as part of the KKLT mechanism of generating a landscape of controlled metastable de Sitter vacua in type IIB string theory. We started by outlining a simple bottom-up 4D EFT argument indicating that models which require an uplift of the order $\delta V \gtrsim m_{\rho}^{2} M_{\mathrm{P}}^{2}$ (such as the simplest version of KKLT) are highly sensitive to the functional form of $\delta V(\rho)$ with $\rho$ being the lightest modulus. We have argued that with our current knowledge of nonperturbative volume stabilization and $\overline{D 3}$-brane potentials, the existence of SUSYbreaking de Sitter vacua within the simplest version of KKLT is hard to establish.

It is for this reason that we have turned to a $10 \mathrm{D}$ description of the required nonperturbative effects that is powerful enough to decide the fate of the original version of the KKLT uplift as well as its racetrack improvement.

In order to do so, we have relied on two central methodological steps. First, we were interested in the energetics of the volume modulus in a full 10D description. Assuming a solution to the full 10D system exists, we were interested in the on-shell potential of this solution, and its scaling under changes of the stabilized CY volume. Conveniently this on-shell scalar potential incorporates all geometric backreaction effects by expressing them via the matter contributions to the theory-provided the backreacted solution exists. For this purpose it was crucial that we could determine this on-shell potential from the matter sources alone by combining the trace-reversed 10D Einstein equations with the 10D Bianchi identities [70,72,73].

We have analyzed a simple toy model in 6D consisting of a quantized two-form flux on a positively curved $S^{2}$ and 3-branes, where fully backreacted solutions are easy to establish. We showed that sufficient uplifting by the 3-branes to naively reach de Sitter led to significant flattening of the uplift: by this we mean that the increase of the $S^{2}$ radius $R$ with increasing uplift occurred such that the total vacuum energy remained AdS while approaching zero from below, supporting the 4D EFT worry. Without including the backreaction on $R$ the uplift would have been 
linear in the tension of the 3-branes which would have naïvely allowed for de Sitter minima.

We then extended this toy model by adding a positive 6D cosmological constant. This allowed us to somewhat decouple the scale of the AdS vacuum energy from $m_{\rho}^{2}$ prior to adding the 3-branes for uplifting. In this case, the flattening from volume increase becomes less pronounced, and it is possible to reach de Sitter. From here we concluded that for models where reaching de Sitter requires $\delta V_{\overline{D 3}} \gtrsim$ $m_{\rho}^{2} M_{\mathrm{P}}^{2}$ according to the off-shell potential, the details of the method of volume stabilization are essential.

Based on this, we needed to reanalyze volume stabilization in KKLT itself in 10D. We started by rederiving the backreaction onto the flux geometry caused by the gaugino condensation process on a D7-brane stack in the KKLT setup based on $[70,72,73]$ and extracting for the first time the full contribution from the D7-gaugino condensate to the matter field side of the trace-reversed Einstein equations. This allowed us to calculate an on-shell potential for the $\rho$ modulus in the simplest KKLT setup. In turn, this enabled us to understand the minimization of the $\rho$ potential into a SUSY AdS minimum while matching the step $24 \mathrm{D}$ effective description outlined above. A crucial result of this analysis is that the $(0,3)$ component of the three-form flux generating the small $\left|W_{0}\right|$ VEV of the Gukov-VafaWitten flux superpotential in step 1 becomes effectively localized at the D7-brane stack due to the backreaction to the formation of the gaugino condensation on the 7-branes.

Finally, we added back an anti-D3-brane at the bottom of the throat to complete the KKLT recipe. We observed that the in general complicated field profiles that the antibrane sources in the warped throat fall off too rapidly towards the bulk Calabi-Yau to cause relevant effects in the on-shell potential. In contrast the on-shell potential preserves its functional form but is to be evaluated at the adjusted value of the Calabi-Yau volume and the condensate. In other words we have calculated the on-shell potential for $\rho$ as a function of the anti- $D 3$-induced $\rho$ increase.

Because for a single gaugino condensate arising from a single D7-brane stack the on-shell potential remains manifestly negative (at leading order in an expansion in inverse compactification volume), the 4D vacuum energy flattens out as a function of increasing anti-D3-brane tension sufficiently strongly to prevent a successful uplift to de Sitter. The simplest KKLT vacua are therefore metastable SUSY-breaking AdS vacua always. For sufficiently small warped antibrane tension, the uplift simply adds to the AdS vacuum energy as predicted by the usual off-shell potential. We match this behavior to the effective $4 \mathrm{D}$ description of the anti-D3-brane by a nilpotent superfield $S$ appearing in the superpotential. The flattening of the uplift then requires the presence of an unsuppressed $S\langle\lambda \lambda\rangle$ coupling in the superpotential.

However, we then proceed to replace the single nonperturbative effect driving SUSY stabilization of $\rho$ by a racetrack superpotential arising from a product gauge group with at least two condensing subgroups (on one or several D7-brane stacks). In this case we can tune the supersymmetric AdS vacuum energy before uplifting arbitrarily close to zero, thus breaking the single-condensate KKLT relation $m_{\rho}^{2} M_{\mathrm{P}}^{2} \sim\left|V_{\text {AdS }}\right|$ into $m_{\rho}^{2} M_{\mathrm{P}}^{2} \gg\left|V_{\text {AdS }}\right|$. While this tuning possibility was long known in the context of the effective 4D supergravity description of the gaugino condensates, we provide a 10D description of this situation, and identify the 10D origin of the tuning possibility.

Relying on this tuning, the amount of uplifting $\delta V \sim$ $\left|V_{\text {AdS }}\right| \ll m_{\rho}^{2} M_{\mathrm{P}}^{2}$ is now perturbatively small. We can thus treat $\rho$ as rigid, which seems to make uplifting to de Sitter possible in the racetrack case both from the 4D and 10D perspectives.

Summing up our results, we believe that they enable a 10D understanding of the KKLT mechanism including its racetrack improvement. This relaxes the necessity of relying on the purely $4 \mathrm{D}$ methods employed so far for the steps of volume stabilization and uplifting in the KKLT framework.

\section{ACKNOWLEDGMENTS}

We thank Wilfried Buchmüller, Markus Dierigl, Ben Freivogel, Luis Ibáñez, Fernando Marchesano, Paul Oehlmann, Susha Parameswaran, Thomas Van Riet, Irene Valenzuela, Timo Weigand, and especially Francisco Pedro, Eva Silverstein, and Angel Uranga for various illuminating discussions. We are deeply grateful to Arthur Hebecker and Liam McAllister for carefully reading the manuscript and providing many very useful comments and extensive discussions. We are grateful for the staffs at SITP in Stanford, the IFT UAM/CSIC, and the PASCOS 2017 conference in Madrid, as well as the Lorentz Center Workshop "Theoretical Approaches to Cosmic Acceleration" in Leiden for their warm hospitality during various stages of this work. This work is supported by the ERC Consolidator Grant STRINGFLATION under the HORIZON 2020 Grant Agreement No. 647995.

\section{APPENDIX A: TYPE IIB AND THE NO-GO THEOREM OF GKP}

For flux compactifications of type IIB string theory, the no-go theorem of [67] was extended significantly by Giddings, Kachru,and Polchinski [14]: following them, we start with the Einstein frame action of type IIB supergravity,

$$
\begin{aligned}
S_{\mathrm{IIB}}= & 2 \pi \int_{M_{10}}\left(* R_{10}-\frac{d \tau \wedge * d \bar{\tau}}{2(\operatorname{Im}(\tau))^{2}}-\frac{G_{3} \wedge * \overline{G_{3}}}{2 \operatorname{Im}(\tau)}\right. \\
& \left.-\frac{F_{5} \wedge * F_{5}}{4}-\frac{i C_{4} \wedge G_{3} \wedge \overline{G_{3}}}{4 \operatorname{Im}(\tau)}\right)+S_{\mathrm{loc}}
\end{aligned}
$$

where $\tau=C_{0}+i e^{-\Phi}$ is the axiodilaton, $G_{3}=F_{3}-\tau H_{3}$ is the complexified three-form field strength, and 


$$
F_{5}=d C_{4}-\frac{1}{2} C_{2} \wedge H_{3}+\frac{1}{2} B_{2} \wedge F_{3}
$$

$S^{\text {loc }}$ is the action of localized objects and we have set $(2 \pi)^{2} \alpha^{\prime}=1$.

Let us take the following ansatz for the $10 \mathrm{D}$ metric:

$$
d s^{2}=e^{2 \mathcal{A}(y)} \tilde{g}_{\mu \nu}^{(4)}(x) d x^{\mu} d x^{\nu}+\underbrace{e^{-2 \mathcal{A}(y)} \tilde{g}_{m n}^{(6)}(y)}_{\equiv g_{m n}^{6}(y)} d y^{m} d y^{n},
$$

where $\tilde{g}_{\mu \nu}^{4}$ is a maximally symmetric 4D metric. Then, the most general ansatz for $F_{5}$ that respects the symmetries of the $4 \mathrm{D}$ space-time is

$$
F_{5}=d C_{4}+\mathcal{F}_{5}
$$

where $\mathcal{F}_{5}$ is purely internal and satisfies

$$
d \mathcal{F}_{5}=\frac{i G_{3} \wedge \overline{G_{3}}}{2 \operatorname{Im}(\tau)}+\frac{T_{3}}{2 \pi} \rho_{D 3}^{\mathrm{loc}},
$$

with $D 3$-brane charge $T_{3}=2 \pi$ and $D 3$-charge density of localized objects $\rho_{D 3}^{\text {loc }}$. $C_{4}$ can be expressed as

$$
C_{4}=\alpha(y) \sqrt{-\tilde{g}^{4}} d x^{0} \wedge d x^{1} \wedge d x^{2} \wedge d x^{3}
$$

with a real function $\alpha(y)$ and is subject to the self-duality constraint $* d C_{4}=\mathcal{F}_{5}$.

The 4D components of the 10D Ricci tensor can be expressed as

$$
R_{\mu \nu}=\tilde{R}_{\mu \nu}-\frac{1}{4} g_{\mu \nu} e^{-4 \mathcal{A}} \nabla^{2} e^{4 \mathcal{A}}
$$

where $\tilde{R}_{\mu \nu}$ is the 4D Ricci tensor of the metric $\tilde{g}_{\mu \nu}^{4}$. Therefore, the trace over the 4D components of Einstein's equations can be expressed as

$$
\begin{aligned}
\tilde{R}_{4}-\tilde{\nabla}_{6}^{2} e^{4 \mathcal{A}}= & -\frac{e^{2 \mathcal{A}}}{2 \operatorname{Im}(\tau)}\left|G_{3}\right|^{2}-e^{-6 \mathcal{A}}\left((\partial \alpha)^{2}\right. \\
& \left.+\left(\partial e^{4 \mathcal{A}}\right)^{2}\right)-\frac{1}{8 \pi} e^{2 \mathcal{A}}\left(T_{m}^{m}-T_{\mu}^{\mu}\right)^{\mathrm{loc}}
\end{aligned}
$$

where $T_{M N}^{\text {loc }}=-\frac{2}{\sqrt{-g}} \frac{\delta S_{\text {loc }}}{\delta g^{M N}}$ is the energy-momentum tensor of localized sources and $\tilde{R}_{4 D}$ is the Ricci-scalar of the metric $\tilde{g}^{4}$.

For the ansatz (A6), the $F_{5}$ Bianchi identity can be expressed as

$$
\tilde{\nabla}_{6}^{2} \alpha=\frac{i e^{2 \mathcal{A}}}{2 \operatorname{Im}(\tau)} G_{3} \cdot *_{6} \overline{G_{3}}+2 e^{-6 \mathcal{A}}(\partial \alpha) \cdot\left(\partial e^{4 \mathcal{A}}\right)+\frac{T_{3}}{2 \pi} e^{2 \mathcal{A}} \rho_{3}^{\mathrm{loc}},
$$

and by taking the difference between (A8) and (A9) one obtains $\tilde{\nabla}^{2} \Phi^{-}=\tilde{R}_{4 D}+\frac{e^{2 \mathcal{A}}}{\operatorname{Im}(\tau)}\left|G_{3}^{-}\right|^{2}+e^{-6 \mathcal{A}}\left|\partial \Phi^{-}\right|^{2}+e^{2 \mathcal{A}} \frac{\Delta^{\mathrm{loc}}}{2 \pi}$,

where $\tilde{R}_{4 D}$ is the $4 \mathrm{D}$ Ricci scalar and we have defined

$$
\begin{aligned}
G_{3}^{ \pm} & \equiv \frac{1}{2}\left(*_{6} \pm i\right) G_{3}, \quad \Phi^{ \pm} \equiv e^{4 \mathcal{A}} \pm \alpha, \quad \text { and } \\
\Delta^{\mathrm{loc}} & \equiv \frac{1}{4}\left(T_{m}^{m}-T_{\mu}^{\mu}\right)^{\mathrm{loc}}-T_{3} \rho_{3}^{\mathrm{loc}} .
\end{aligned}
$$

Integrating this expression over the compact manifold, it follows that in the absence of localized sources that violate the BPS-like bound

$$
\Delta^{\mathrm{loc}} \geq 0
$$

the $4 \mathrm{D}$ vacuum energy $V \cdot M_{\mathrm{P}}^{-4}=\frac{1}{4} M_{\mathrm{P}}^{-2} \tilde{R}_{4 D}$ is negative semidefinite,

$V \cdot M_{\mathrm{P}}^{-4}=\int \frac{d^{6} y \sqrt{g^{6}}}{16 \pi \mathcal{V}_{w} \tilde{\mathcal{V}}_{w}}\left[-e^{8 \mathcal{A}} \frac{\Delta}{2 \pi}-\left|\partial \Phi^{-}\right|^{2}\right] \leq 0$,

where

$$
\begin{aligned}
\Delta & \equiv 2 \pi \frac{\left|G_{3}^{-}\right|^{2}}{\operatorname{Im}(\tau)}+\Delta^{\mathrm{loc}}, \quad \tilde{\mathcal{V}}_{w} \equiv \int_{M_{6}} d^{6} y \sqrt{g^{6}} e^{6 \mathcal{A}}, \quad \text { and } \\
\mathcal{V}_{w} & \equiv \int_{M_{6}} d^{6} y \sqrt{g^{6}} e^{2 \mathcal{A}} .
\end{aligned}
$$

In this notation the solutions of [14] correspond to

$$
G_{3}^{-}=\Delta^{\mathrm{loc}}=\tilde{R}_{4 D}=\Phi^{-}=0 .
$$

It is important to note that these solutions leave at least one modulus unfixed, the volume modulus. Clearly the inclusion of any further sources of positive energy cannot lead to a stable solution but rather lead to decompactification.

This is true in particular for $\overline{D 3}$-branes: the fact that they give a negative contribution to $\Delta^{\text {loc }}$ has the following simple interpretation: without Kähler moduli stabilization the presence of an $\overline{D 3}$-brane leads to decompactification.

\section{APPENDIX B: CONTRIBUTIONS OF FLUXES AND LOCALIZED OBJECTS TO THE ON-SHELL POTENTIAL}

In this appendix we would like to summarize the contributions of localized objects of tension $T_{p}$ and $p$-form fluxes to the on-shell potential (27) following [67].

\section{The contribution of $\boldsymbol{p}$-form fluxes}

For definiteness, we consider the case of a higherdimensional $p$-form field strength, with action 


$$
S_{p-\text { form }}=\frac{M^{D-2}}{2} \int\left(-\frac{1}{2} F_{p} \wedge * F_{p}\right) .
$$

Then, the energy-momentum tensor reads

$T_{M N}=\frac{1}{2} \frac{1}{(p-1) !} F_{M O_{2} \cdots O_{p}} F_{N} O_{2} \cdots O_{p}-\frac{1}{4} g_{M N}\left|F_{p}\right|^{2}$.

Let us now write $F_{p}=F_{p \text {,ext }}+F_{p \text {,int }}$, where $F_{p \text {,ext }}$ threads the four noncompact directions if $p \geq 4$ and vanishes if $p<4$ while $F_{p \text {,int }}$ threads internal directions only. It follows that

$$
\begin{aligned}
T_{P}^{P} & =\frac{2 p-D}{4}\left(\left|F_{p, \text { ext }}\right|^{2}+\left|F_{p, \text { int }}\right|^{2}\right) \quad \text { and } \\
T_{\mu}^{\mu} & =\left|F_{p, \text { ext }}\right|^{2}-\left|F_{p, \text { int }}\right|^{2}
\end{aligned}
$$

and the on-shell potential (27) is proportional to a (weighted) integral over the expression

$$
\begin{aligned}
& (D-6) T_{\mu}^{\mu}-4 T_{m}^{m} \\
& \quad=2(D-p-1) \underbrace{\left|F_{p, \text { ext }}\right|^{2}}_{\leq 0}+2(1-p) \underbrace{\left|F_{p, \text { int }}\right|^{2}}_{\geq 0} .
\end{aligned}
$$

It follows that internal components make a positive contribution only in the case $p=0$ and do not contribute when $p=1$ [67]. External components make a positive contribution for the top-form $p=D$ and a vanishing contribution for $p=D-1$. The latter two are of course equivalent to the former two by Hodge duality.

\section{The contribution of localized objects}

In addition, let us see how localized objects of spatial dimension $p$ and tension $T_{p}$ contribute. For this, consider a DBI-like action

$$
S_{\mathrm{loc}}=-T_{p} \int d^{p+1} \xi \sqrt{-\operatorname{det}(P[g])},
$$

where $P[g]$ is the pullback of the ambient space metric on the localized objects world volume. The energy-momentum tensor is

$$
T_{M N}=-\frac{T_{p}}{M^{D-2}} \Pi_{M N}(\Sigma) \cdot \delta(\Sigma),
$$

where $\Pi(\delta)$ is the projector on the $p-3$ cycle $\Sigma$ that the object wraps and $\delta(\Sigma)$ is the transverse $\delta$ function. Hence, they contribute with

$$
(D-6) T_{\mu}^{\mu}-4 T_{m}^{m}=-4(D-p-3) \frac{T_{p}}{M^{D-2}} \delta(\Sigma) .
$$

For positive tension $T_{p}$ one immediately sees that positive contributions to the on-shell potential (27) come only from space time filling or codimension one objects. Also, negative tension objects of spatial dimension smaller than $D-3$ give a positive contribution, while codimension two objects do not contribute directly.

\section{APPENDIX C: GAUGINO CONDENSATES AND THE COSMOLOGICAL CONSTANT}

In this appendix we would like to provide the detailed derivation of the formulas used in Sec. IV. The perturbed $G_{3}$ profile has previously been calculated in [72]. We provide also the contribution of all the terms under the integrand of (27).

We are interested in the effects of the gaugino condensate in the bulk Calabi-Yau where (by definition) the backreaction of fluxes on the geometry is negligible. Thus, in the following we work in the constant warp factor approximation where $R_{m n}=0$. Hence, all results are valid to leading order in an inverse volume expansion.

\section{The $\bar{\lambda} \bar{\lambda} G_{3}$ coupling}

We will follow Appendix A of [72] using results of Appendix A of [94]. For vanishing world volume flux, the fermionic part of the $\kappa$-symmetric $D 7$-brane action reads [95-97]

$S_{D 7}^{\mathrm{ferm}}=i \pi \int_{\Sigma_{8}} e^{-\phi} \sqrt{-\operatorname{det} g} \bar{\theta}(1-\Gamma)\left(\Gamma^{\alpha} \mathcal{D}_{\alpha}-\frac{1}{2} \mathcal{O}\right) \theta$,

where $\alpha, \beta \ldots$ are $8 d$ indices and $\theta$ is the two-component Green-Schwarz (GS) spinor. The operators $\mathcal{D}_{\alpha}$ and $\mathcal{O}$ are the pullbacks of the $10 \mathrm{D}$ operators

$$
\begin{aligned}
& \mathcal{D}_{M} \theta= \nabla_{M} \theta+\frac{1}{4} \underline{H}_{M}\left(\begin{array}{cc}
1 & 0 \\
0 & -1
\end{array}\right) \theta \\
&+\frac{1}{16} e^{\phi}\left(\begin{array}{cc}
0 & \underline{F} \Gamma_{M} \\
-\sigma(\underline{F}) \Gamma_{M} & 0
\end{array}\right) \theta, \\
& \mathcal{O} \theta=\underline{\partial} \phi \theta+\frac{1}{2} \underline{H}\left(\begin{array}{cc}
1 & 0 \\
0 & -1
\end{array}\right) \theta \\
&+\frac{1}{16} e^{\phi}\left(\begin{array}{cc}
0 & \Gamma^{M} \underline{F} \Gamma_{M} \\
-\Gamma^{M} \sigma(\underline{F}) \Gamma_{M} & 0
\end{array}\right) \theta,
\end{aligned}
$$

where $\Gamma_{M}$ are 10D gamma matrices. Furthermore,

$$
\Gamma=-\frac{i \sigma_{2}}{\sqrt{-g}} \frac{\epsilon_{\alpha_{0} \ldots \alpha_{7}}}{8 !} \Gamma^{\alpha_{0} \ldots \alpha_{7}},
$$

and the map $\sigma$ reverses the order of indices. The barred spinor is defined as $\bar{\theta}=\theta^{\dagger} \Gamma^{0}$. Underlined tensors are contracted with gamma matrices. The action $(\mathrm{C} 1)$ is written 
in the (RR-)democratic formulation of type IIB supergravity. This means that $F=F_{1}+\cdots+F_{9}$ and the equations of motion have to be supplemented by the duality constraint

$$
\underline{F} \Gamma_{*}=\underline{F},
$$

in particular $\underline{F}_{7} \Gamma_{*}=\underline{F}_{3}$. Equivalently, one may work with $F_{1}, F_{3}$, and $F_{5}$ only, while doubling the contribution of $F_{1}$ and $F_{3}$ in (C2) and (C3). In this case one only imposes the usual self-duality constraint $\underline{F}_{5} \Gamma_{*}=\underline{F}_{5}$. We will do so in the following.

Imposing the $\kappa$-fixing gauge

$$
\bar{\theta} \Gamma=-\bar{\theta},
$$

and keeping only three-form fluxes with purely internal components, the action can be written as

$$
S_{D 7}^{\mathrm{ferm}}=2 \pi i \int d^{8} \sigma e^{-\phi} \sqrt{-g}\left(\bar{\theta} \Gamma^{\alpha}\left(\nabla_{\alpha}-\frac{1}{2} \partial_{\alpha} \phi\right) \theta+\bar{\theta}\left[\frac{1}{4}\left(\Gamma^{\alpha} \underline{H}_{\alpha}-\underline{H}\right) \sigma_{3}+\frac{e^{\phi}}{8}\left(\Gamma^{\alpha} \underline{F}_{3} \Gamma_{\alpha}-\frac{1}{2} \Gamma_{M} \underline{F}_{3} \Gamma^{M}\right) \sigma_{1}\right] \theta\right) .
$$

This action is transformed to a $10 \mathrm{D}$ Einstein frame using

$$
g_{\alpha \beta} \rightarrow e^{\phi / 2} g_{\alpha \beta}, \quad \Rightarrow \Gamma^{M} \rightarrow e^{-\phi / 4} \Gamma^{M},
$$

and the redefinition

$$
\Theta \rightarrow e^{-3 \phi / 8} \Theta
$$

The result is

$$
S_{D 7}^{\mathrm{ferm}}=2 \pi i \int d^{8} \sigma \sqrt{-g} \bar{\theta} \Gamma^{\alpha} \nabla_{\alpha} \theta+e^{\phi / 2} \bar{\theta}\left[\frac{e^{-\phi}}{4}\left(\Gamma^{\alpha} \underline{H}_{\alpha}-\underline{H}\right) \sigma_{3}+\frac{1}{8}\left(\Gamma^{\alpha} \underline{F}_{3} \Gamma_{\alpha}-\frac{1}{2} \Gamma_{M} \underline{F}_{3} \Gamma^{M}\right) \sigma_{1}\right] \theta .
$$

Let us further massage this coupling of the schematic form $\bar{\theta} \theta G_{3}$. Two expressions need to be simplified,

$$
\begin{gathered}
\mathrm{I}: \Gamma^{\alpha} \underline{H}_{\alpha}-\underline{H}, \\
\mathrm{II}: \Gamma^{\alpha} \underline{F}_{3} \Gamma_{\alpha}-\frac{1}{2} \Gamma_{M} \underline{F}_{3} \Gamma^{M} .
\end{gathered}
$$

Let us decompose the three-form fluxes as follows:

$$
\begin{aligned}
& F_{3}=F_{3}^{(0)}+F_{3}^{(1)}+F_{3}^{(2)}, \\
& H_{3}=H_{3}^{(0)}+H_{3}^{(1)}+H_{3}^{(2)},
\end{aligned}
$$

where the upstairs index denotes the number of indices transverse to the divisor $\Sigma_{4}$ the 7-brane wraps. By commuting $\Gamma$ matrices one can show that

$$
\begin{aligned}
& \Gamma^{\alpha} \underline{F} \Gamma_{\alpha}-\frac{1}{2} \Gamma_{M} \underline{F} \Gamma^{M} \\
& \quad=-2 \frac{F_{i j k}}{3 !}\left(P_{\perp}\left(\Gamma^{i}\right) \Gamma^{j} \Gamma^{k}+\Gamma^{i} P_{\perp}\left(\Gamma^{j}\right) \Gamma^{k}+\Gamma^{i} \Gamma^{j} P_{\perp}\left(\Gamma^{k}\right)\right) .
\end{aligned}
$$

Here, $P_{\perp}\left(\Gamma^{i}\right)=\Gamma^{i}$ if the index $i$ is transverse to $\Sigma_{4}$ while it vanishes otherwise. Under the decomposition (C13) this reads

$$
\Gamma^{\alpha} \underline{F} \Gamma_{\alpha}-\frac{1}{2} \Gamma_{M} \underline{F} \Gamma^{M}=-2 \underline{F}^{(1)}-4 \underline{F}^{(2)} .
$$

Similarly, one shows that

$$
\Gamma^{\alpha} \underline{H}_{\alpha}-\underline{H}=2 \underline{H}-3 \frac{H_{i j k}}{3 !} P_{\perp}\left(\Gamma^{i}\right) \Gamma^{j} \Gamma^{k}=2 \underline{H}^{(0)}+\underline{H}^{(1)} .
$$

Thus, the $\bar{\theta} \theta G_{3}$ coupling reads

$$
\begin{aligned}
S_{D 7} \supseteq & \frac{\pi}{2} i \int d^{8} \sigma \sqrt{-g} e^{\phi / 2} \bar{\theta}\left(e^{-\phi}\left(2 \underline{H}^{(0)}+\underline{H}^{(1)}\right) \sigma_{3}\right. \\
& \left.-\left(\underline{F}^{(1)}+2 \underline{F}^{(2)}\right) \sigma_{1}\right) \theta .
\end{aligned}
$$

To perform the dimensional reduction one decomposes the 10D $\Gamma$-matrices as

$$
\Gamma^{\mu}=e^{-\mathcal{A}} \tilde{\gamma}^{\mu} \otimes \mathbb{1}, \quad \mu=0, \ldots, 3,
$$




$$
\Gamma^{i}=\tilde{\gamma}_{*} \otimes \gamma_{6 D}^{i-3}, \quad i=4, \ldots, 9
$$

with $4 \mathrm{D}$, respectively $6 \mathrm{D}$, gamma matrices $\tilde{\gamma}^{\mu}$ and $\gamma_{6 D}^{i-3}$.

The dimensional reduction ansatz for the gaugino is

$$
\begin{aligned}
& \theta_{1}=\frac{1}{4 \pi} e^{-2 \mathcal{A}} \lambda_{D} \otimes \eta_{1}+\text { c.c. }, \\
& \theta_{2}=-\frac{1}{4 \pi} e^{-2 \mathcal{A}} \lambda_{D} \otimes \eta_{2}+\text { c.c. }
\end{aligned}
$$

Here, $\lambda_{D}$ is a $4 \mathrm{D}$ Dirac spinor of positive chirality, and c.c. denotes charge conjugation. Moreover, $\eta_{1}$ and $\eta_{2}$ are the $6 \mathrm{D}$ Weyl spinors that appear in the 10D SUSY parameters:

$$
\epsilon_{1}=\xi \otimes \eta_{1}+\text { c.c., } \quad \epsilon_{2}=\xi \otimes \eta_{2}+\text { c.c. }
$$

For $03 / 07$ orientifolds with ISD fluxes and $D 3 / D 7$-branes, the relation between $\eta \equiv \eta_{1}$ and $\eta_{2}$ is [69]

$$
\eta_{2}=i \eta_{1}
$$

Thus, we may write $\theta_{1}=\psi+\psi^{c}, \quad \theta_{2}=-i\left(\psi-\psi^{c}\right), \quad \psi \equiv \frac{1}{4 \pi} e^{-2 \mathcal{A}} \lambda_{D} \otimes \eta$,

where the superscript ${ }^{c}$ denotes charge conjugation.

With this one calculates that

$$
\begin{aligned}
e^{4 \mathcal{A}} \bar{\theta} \Gamma_{i j k} \sigma_{3} \theta & =2 e^{4 \mathcal{A}} \overline{\psi^{c}} \Gamma_{i j k} \psi-\text { c.c. } \\
& =-\frac{i}{8 \pi^{2}} \overline{\lambda_{D}^{c}} \lambda_{D}\left(\eta^{c}\right)^{\dagger} \gamma_{i j k} \eta-\text { c.c. }
\end{aligned}
$$

and

$$
\begin{aligned}
e^{4 \mathcal{A}} \bar{\theta} \Gamma_{i j k} \sigma_{1} \theta & =-2 i e^{4 \mathcal{A}} \overline{\psi^{c}} \Gamma_{i j k} \psi-\text { c.c. } \\
& =-\frac{1}{8 \pi^{2}} \overline{\lambda_{D}^{c}} \lambda_{D}\left(\eta^{c}\right)^{\dagger} \gamma_{i j k} \eta-\text { c.c. }
\end{aligned}
$$

Here, the 4D barred spinor is the usual Dirac conjugate $\overline{\lambda_{D}}=i \lambda_{D}^{\dagger} \tilde{\gamma}^{0}$.

Let us define

$$
\begin{aligned}
G_{3}^{(i, j)} & :=F_{3}^{(i)}-i e^{-\phi} H_{3}^{(j)}, \\
\mathcal{G}_{3} & :=G_{3}^{(1,1)}+2 G_{3}^{(2,0)} .
\end{aligned}
$$

Then we may write the result as

$$
\begin{aligned}
S_{D 7} & \supseteq \int_{M_{10}} d^{10} x \sqrt{-g} \mathcal{L}_{\lambda \lambda}^{\mathrm{loc}} \equiv \int_{M_{4} \times \Sigma_{4}} d^{8} \sigma \sqrt{-g} e^{-4 \mathcal{A}} e^{\phi / 2} \frac{i \overline{\lambda_{D}^{c}} \lambda_{D}}{16 \pi} \Omega \cdot \mathcal{G}_{3}+\text { c.c. } \\
& =\int_{M_{4} \times \Sigma_{4}} d^{8} \sigma \sqrt{-g} e^{-4 \mathcal{A}} e^{\phi / 2} \frac{\bar{\lambda}_{\dot{\alpha}} \bar{\lambda}^{\dot{\alpha}}}{16 \pi} \Omega \cdot \mathcal{G}_{3}+\text { c.c. }=\int_{M_{10}} \delta_{D}^{(0)} e^{-4 \mathcal{A}} e^{\phi / 2} \frac{\bar{\lambda}_{\dot{\alpha}} \bar{\lambda}^{\dot{\alpha}}}{16 \pi} \mathcal{G}_{3} \wedge * \Omega+\text { c.c. }
\end{aligned}
$$

where we have also used that the holomorphic three-form $\Omega$ is given in terms of the $6 \mathrm{D}$ spinor $\eta$ as

$$
\Omega_{i j k}=\left(\eta^{c}\right)^{\dagger} \gamma_{i j k} \eta,
$$

and we have written the action as a 10D integral by introducing the scalar delta-function $\delta_{D}^{(0)}$ that localizes on the divisor $\Sigma_{4}$. It satisfies [70]

$$
2 \pi \delta_{D}^{(0)}=\nabla^{2} \operatorname{Re} \log h=2 g^{i \bar{i}} \nabla_{i} \nabla_{\bar{i}} \operatorname{Re} \log h,
$$

where the holomorphic function $h$ defines the divisor that the 7-brane wraps via $h=0$.

Moreover, in the second line of (C29) we have chosen the 4D Weyl representation where the spinor $\lambda_{D}$ takes the form $\left(0, \bar{\lambda}^{\dot{\alpha}}\right)^{T}$. Finally, we used that $\overline{\lambda_{D}^{c}} \lambda_{D}=\lambda_{D}^{T} C \lambda_{D}=$ $-i \bar{\lambda}_{\dot{\alpha}} \bar{\lambda}^{\dot{\alpha}}$. From now on we will write $\bar{\lambda} \bar{\lambda}=\bar{\lambda}_{\dot{\alpha}} \bar{\lambda}^{\dot{\alpha}}$ and $\lambda \lambda=(\bar{\lambda} \bar{\lambda})^{*}$.
One further simplification can be made: $\Omega \cdot \mathcal{G}_{3}$ projects on the $(0,3)$ piece of $\mathcal{G}_{3}$. Because the submanifold that the 7-branes wrap is complex [98,99] (see also [100] for flux compactifications beyond GKP), the components of $F_{3}$ and $\mathrm{H}_{3}$ that have precisely zero or two legs transverse to the brane are of Hodge type $(2,1) \oplus(1,2)$ which implies that $\mathcal{G}_{3} \cdot \Omega=G_{3} \cdot \Omega$. Thus, we finally arrive at

$$
S_{D 7} \supseteq \int_{M_{10}} \pi \delta_{D}^{(0)} e^{\phi / 2} e^{-4 \mathcal{A}} \frac{\bar{\lambda} \bar{\lambda}}{16 \pi^{2}} G_{3} \wedge * \Omega+\text { c.c.. }
$$

\section{The $G_{3}$ equations of motion and their solution}

In the presence of a nonvanishing expectation value $\langle\lambda \lambda\rangle$, the equations of motion and Bianchi identity of $G_{3}$ read 
$d \Lambda-\frac{d \tau}{\tau-\bar{\tau}} \wedge(\Lambda+\bar{\Lambda})=d X-\frac{d \tau}{\tau-\bar{\tau}} \wedge(X+\bar{X})$,

$d G^{+}-\frac{d \tau}{\tau-\bar{\tau}} \wedge\left(G^{+}+\bar{G}^{+}\right)=d G^{-}-\frac{d \tau}{\tau-\bar{\tau}} \wedge\left(G^{-}+\bar{G}^{-}\right)$,

where $\Lambda \equiv e^{4 \mathcal{A}} *_{6} G_{3}-i \alpha G_{3}$ and $X$ is defined via

$$
\begin{aligned}
d^{4} x & \wedge\left(d X-\frac{d \tau}{\tau-\bar{\tau}} \wedge(X+\bar{X})\right) \\
& =-\frac{i}{2 \pi}\left(\tau d\left(\frac{\partial \mathcal{L}_{\lambda \lambda}^{\mathrm{loc}}}{\partial d C_{2}}\right)+d\left(\frac{\partial \mathcal{L}_{\lambda \lambda}^{\mathrm{loc}}}{\partial d B_{2}}\right)\right),
\end{aligned}
$$

which determines

$$
X=e^{-\phi / 2} \frac{\langle\lambda \lambda\rangle}{16 \pi^{2}} \delta_{D} *_{6} \bar{\Omega} .
$$

The ISD solutions correspond to $\Lambda=e^{4 \mathcal{A}} G^{-}=0$.

We expand the above equation to linear order in $\langle\lambda \lambda\rangle$. Equation (A10) implies that to this order $\Phi^{-}=0$. Therefore,

$\Lambda=\frac{1}{2}\left(\Phi^{+} G_{3}^{-}+\Phi^{-} G_{3}^{+}\right)=e^{4 \mathcal{A}} G_{3}^{-}+\mathcal{O}\left(|\langle\lambda \lambda\rangle|^{2}\right)$,

and hence

$$
d\left(e^{4 \mathcal{A}} G_{3}^{-}\right)=d X
$$

Note that it is not possible to simply set $e^{4 \mathcal{A}} G_{3}=X$ because by definition $G_{3}^{-}$is IASD while $X$ is ISD. In the flat Calabi-Yau limit $R_{i j}=0$ the solution was derived in [70]. It is shown that bulk IASD fluxes of Hodge type $(1,2)$ are sourced:

$$
\begin{aligned}
\left(e^{4 \mathcal{A}} G_{3}^{-}\right)_{i \bar{j} \bar{k}} & =-\frac{i}{\pi} e^{-\phi / 2} \frac{\langle\lambda \lambda\rangle}{16 \pi^{2}}\left(\nabla_{i} \nabla_{l} \operatorname{Re}(\log h)\right) g^{l \bar{m}} \bar{\Omega}_{\bar{m} \bar{j} \bar{k}}, \\
\Rightarrow 2 \pi e^{\phi}\left|G_{3}^{-}\right|^{2} & =\frac{16}{\pi} e^{-8 \mathcal{A}}\left|\frac{\langle\lambda \lambda\rangle}{16 \pi^{2}}\right|^{2}\left|\nabla_{i} \nabla_{j} \operatorname{Re} \log h\right|^{2},
\end{aligned}
$$

using $|\Omega|^{2} \equiv \frac{1}{3 !} \Omega_{i j k} \bar{\Omega}^{i j k}=8$. Equation (C33) only determines the IASD flux. The ISD part of $G_{3}$ follows from imposing the Bianchi identity (C34), i.e.

$$
d G_{3}^{+}=d G_{3}^{-}=e^{-4 \mathcal{A}} d X,
$$

which is solved by

$e^{4 \mathcal{A}} G_{3}^{+}=X=\frac{1}{\pi} e^{-\phi / 2} \frac{\langle\lambda \lambda\rangle}{16 \pi^{2}}\left(g^{i \bar{j}} \nabla_{i} \nabla_{\bar{j}} \operatorname{Re}(\log h)\right) \bar{\Omega}$.

This localized ISD flux was computed in [72].

\section{The contributions to the on-shell potential}

We are now ready to derive the contribution of the fermionic action (C1) to $\Delta_{\text {loc }}$. For this one has to vary the action with respect to the vielbein $e_{a}^{M}$ defined by

$$
g^{M N}=e_{a}^{M} e_{b}^{N} \eta^{a b} .
$$

There are two contributions: the first is due to the variation of the volume form. Because the $D 7$ has codimension two, this will drop out in $\Delta_{\text {loc }}$. The second contribution comes from varying the curved space $\Gamma$-matrices $\Gamma^{M}=e_{a}^{M} \Gamma^{a}$ with constant matrices $\Gamma^{a}$. Then, by using $g^{m n} \frac{\partial}{\partial g^{m n}}=\frac{1}{2} e_{c}^{m} \frac{\partial}{\partial e_{c}^{m}}$ it is straightforward to obtain the desired negative contribution to $\Delta_{\text {loc }}$,

$$
\begin{aligned}
\Delta_{\mathrm{loc}} \supseteq \Delta_{\mathrm{loc}}^{\lambda \lambda} & \equiv-\frac{1}{4} e_{c}^{m} \frac{\partial \mathcal{L}_{\lambda \lambda}^{\mathrm{loc}}}{\partial e_{c}^{m}} \\
& =-\frac{3 \pi}{4} e^{\phi / 2} e^{-4 \mathcal{A}} \frac{\langle\bar{\lambda} \bar{\lambda}\rangle}{16 \pi^{2}} \Omega \cdot G_{3} \delta_{D}^{(0)}+\text { c.c. } \\
& =-\frac{12}{\pi} e^{-8 \mathcal{A}}\left|\frac{\langle\lambda \lambda\rangle}{16 \pi^{2}}\right|^{2}\left|g^{i j} \nabla_{i} \nabla_{\bar{j}} \operatorname{Re}(\log h)\right|^{2}<0 .
\end{aligned}
$$

Bulk IASD fluxes (C39) contribute to $\Delta$ with

$$
\Delta_{\text {bulk }}^{\lambda \lambda} \equiv \frac{16}{\pi} e^{-8 \mathcal{A}}\left|\frac{\langle\lambda \lambda\rangle}{16 \pi^{2}}\right|^{2}\left|\nabla_{i} \nabla_{j} \operatorname{Re} \log h\right|^{2}>0
$$

Thus finally,

$$
\begin{aligned}
\Delta \supseteq \Delta^{\lambda \lambda} \equiv & \frac{4}{\pi} e^{-8 \mathcal{A}}\left|\frac{\langle\lambda \lambda\rangle}{16 \pi^{2}}\right|^{2}\left[4\left|\nabla_{i} \nabla_{j} \operatorname{Re} \log h\right|^{2}\right. \\
& \left.-3\left|g^{i \bar{i}} \nabla_{i} \nabla_{\bar{i}} \operatorname{Re} \log h\right|^{2}\right] .
\end{aligned}
$$

For a set of $n$ stacks of 7-branes that wrap divisors $\Sigma_{a}$, $a=1, \ldots, n$, the contribution to $\Delta$ becomes

$$
\begin{aligned}
\Delta^{\lambda \lambda}= & \frac{4}{\pi} e^{-8 \mathcal{A}}\left[4\left|\sum_{a=1}^{n} \frac{\langle\lambda \lambda\rangle_{a}}{16 \pi^{2}} \nabla_{i} \nabla_{j} \operatorname{Re} \log h_{a}\right|^{2}\right. \\
& \left.-3 \sum_{a=1}^{n}\left|\frac{\langle\lambda \lambda\rangle_{a}}{16 \pi^{2}} g^{i \bar{i}} \nabla_{i} \nabla_{\bar{i}} \operatorname{Re} \log h_{a}\right|^{2}\right]
\end{aligned}
$$

Because the contribution to the cosmological constant is determined by integrating this expression over the internal manifold, we may partially integrate 


$$
\begin{aligned}
\int_{M_{6}} *_{6}\left|\nabla_{i} \nabla_{j} g\right|^{2} & =\int_{M_{6}} *_{6} g^{i \bar{i}} g^{j \bar{j}} \nabla_{i} \nabla_{j} g \nabla_{\bar{i}} \nabla_{\bar{j}} g \stackrel{p . I .}{=}-\int_{M_{6}} *_{6}\left(g^{j \bar{j}}\left(g^{i \bar{i}} \nabla_{i} \nabla_{\bar{i}} \nabla_{j} g\right) \nabla_{\bar{j}} g\right) \\
& =-\int_{M_{6}} *_{6}\left(g^{j \bar{j}} g^{\bar{i} i}(\nabla_{j} \nabla_{i} \nabla_{\bar{i}} g+\frac{1}{2} \underbrace{\left[\nabla^{2}, \nabla_{j}\right] g}_{=R_{j k} \partial^{k} g=0}) \nabla_{\bar{j}} g\right) \stackrel{p . I .}{=} \int_{M_{6}} *_{6}\left|g^{i \bar{i}} \nabla_{i} \nabla_{\bar{i}} g\right|^{2} .
\end{aligned}
$$

for any real function $g$.

Therefore, we may write

$$
\Delta^{\lambda \lambda}=\frac{4}{\pi} e^{-8 \mathcal{A}}\left[4\left|\sum_{a=1}^{n} \frac{\langle\lambda \lambda\rangle_{a}}{16 \pi^{2}} \nabla_{i} \nabla_{j} \operatorname{Re} \log h_{a}\right|^{2}-3 \sum_{a=1}^{n}\left|\frac{\langle\lambda \lambda\rangle_{a}}{16 \pi^{2}} \nabla_{i} \nabla_{j} \operatorname{Re} \log h_{a}\right|^{2}\right] .
$$

Crucially, for a single gaugino condensate

$$
\Delta^{\lambda \lambda}=\frac{4}{\pi} e^{-8 \mathcal{A}}\left|\frac{\langle\lambda \lambda\rangle}{16 \pi^{2}} \nabla_{i} \nabla_{j} \operatorname{Re} \log h\right|^{2}>0
$$

which implies that the 4D potential energy is negative and given by

$V \cdot M_{\mathrm{P}}^{-4}=-\frac{1}{8 \pi^{3} \mathcal{V}_{w} \tilde{\mathcal{V}}_{w}} \int d^{6} y \sqrt{g}\left|\frac{\langle\lambda \lambda\rangle}{16 \pi^{2}} \nabla_{i} \nabla_{j} \operatorname{Re} \log h\right|^{2}$.

However, for two condensates the first term in (C48) can be made small by giving the condensates opposite phases. In this case the two terms in (C48) cancel each other at least partially. Of course, for a SUSY vacuum the final result can never be positive but we find it very conceivable that a small SUSY-breaking source (like an $\overline{\mathrm{D} 3}$-brane) can lift such a vacuum to de Sitter.

\section{APPENDIX D: A VOLUME SUPPRESSED CONTRIBUTION}

Here we would like to address an example of a volume suppressed contribution to the on-shell potential (47) that we must neglect for consistency of our expansion scheme. Before uplifting, the flux configuration is as follows:

$$
G_{3}=\underbrace{G_{3}^{(2,1)}}_{\text {harmonic }}+\underbrace{G_{3}^{\lambda \lambda}}_{\text {particular solution }},
$$

where $G_{3}^{\lambda \lambda}$ is the profile that has been computed in [70,72]. Therefore, the localized $(0,3)$ piece in $G_{3}^{\lambda \lambda}$ is in the same cohomology class as the harmonic $G_{3}$ piece of Hodge type $(0,3)$ that would have existed without nonperturbative stabilization. The value of the gaugino condensate is tied to the profile $G_{3}^{\lambda \lambda}$ via the equations of motion. Thus, the condensate will dynamically find its correct value such that the localized $(0,3)$ piece in $G_{3}^{\lambda \lambda}$ can account for all of $W_{0}=\int G_{3} \wedge \Omega$.
We start with this supersymmetric situation and deform it by including a SUSY-breaking source at the bottom of a warped throat. Such a source will push the value of the volume modulus towards larger values and thereby reduce the magnitude of the condensate.

$$
\langle\lambda \lambda\rangle_{0} \rightarrow\langle\lambda \lambda\rangle_{1}
$$

Because the field profile $G_{3}^{\lambda \lambda}$ is still tied to the value of the condensate, in order for this to be consistent with flux quantization and a negligible pull on the complex structure moduli there must develop a harmonic $(0,3)$ piece in $G_{3}$,

$$
G_{3} \rightarrow G_{3}+g^{(0,3)} \bar{\Omega},
$$

such that $\int G_{3} \wedge \frac{\Omega}{\|\Omega\|}$ remains constant. Using the identities

$$
\begin{aligned}
\|\Omega\|^{2} & =\int_{M_{6}} \Omega \wedge \bar{\Omega}=8 \mathcal{V} \\
\text { and } \int_{M_{6}} \delta_{D} \Omega \wedge \bar{\Omega} & =8 \operatorname{Vol}\left(\Sigma_{4}\right),
\end{aligned}
$$

this fixes $g^{(0,3)}$ to be

$g^{(0,3)}=\left.e^{-4 \mathcal{A}}\right|_{\Sigma} e^{-\phi / 2} \frac{\operatorname{Vol}(\Sigma)_{1}}{\mathcal{V}_{1}}\left(\frac{\langle\lambda \lambda\rangle_{0}}{16 \pi^{2}} \frac{\operatorname{Vol}(\Sigma)_{0}}{\operatorname{Vol}(\Sigma)_{1}} \sqrt{\frac{\mathcal{V}_{1}}{\mathcal{V}_{0}}}-\frac{\langle\lambda \lambda\rangle_{1}}{16 \pi^{2}}\right)$.

Here the subindices 0 and 1 denote preuplift, respectively postuplift, situations. Clearly this constant flux piece contributes to $\Delta^{\text {loc }}$ and therefore contributes a term in the on-shell potential (27). However, to leading order in the inverse volume expansion this term must be neglected. This is clear intuitively because only the value of $G_{3}^{(0,3)}$ at the position of the 7-brane divisor $\Sigma_{4}$ enters the on-shell potential (47). If part of the localized term is traded for a constant term to leading order in the inverse transverse volume, only a reduction in the localized $G_{3}^{(0,3)}$ component 
is seen. Let us show this explicitly: the constant flux contributes a term to the on-shell potential

$$
\begin{aligned}
V_{g^{(0,3)}} \cdot M_{\mathrm{P}}^{-4} \equiv & \frac{3}{16 \pi} \frac{\operatorname{Vol}(\Sigma)_{1}^{2}}{\mathcal{V}_{1}^{3}} \frac{\langle\bar{\lambda} \bar{\lambda}\rangle_{1}}{16 \pi^{2}} \\
& \times\left[\frac{\langle\lambda \lambda\rangle_{0}}{16 \pi^{2}} \frac{\operatorname{Vol}(\Sigma)_{0}}{\operatorname{Vol}(\Sigma)_{1}} \sqrt{\frac{\mathcal{V}_{1}}{\mathcal{V}_{0}}}-\frac{\langle\lambda \lambda\rangle_{1}}{16 \pi^{2}}\right]+\text { c.c. }
\end{aligned}
$$

Because all volumes are pushed towards larger values and the value of the condensate decreases exponentially with the volume, we have $\langle\lambda \lambda\rangle_{1}<\langle\lambda \lambda\rangle_{0}$. Hence this contribution is positive.

If a warped tension $\delta V / M_{\mathrm{P}}^{4}=\frac{e^{4 \mathcal{A}_{0}} T_{3}}{(4 \pi)^{2} \mathcal{V}^{2}}$ is added to the setup, it is clear that if the warp factor $e^{\mathcal{A}_{0}}$ is sufficiently small, the change in potential is given precisely by this term. Using this information and our knowledge about the off-shell potential we can deduce how far the volume modulus shifts. We assume the usual exponential dependence

$$
|\langle\lambda \lambda\rangle| \sim e^{-a \operatorname{Vol}(\Sigma)},
$$

and choose to calculate to leading order in $1 / \operatorname{Vol}(\Sigma)$. In this approximation we need only consider the shift of $\langle\lambda \lambda\rangle$ itself and not of explicit volume powers that appear in the above formulas. The shift in the potential in Eq. (57) induced by a small shift $\delta \operatorname{Vol}(\Sigma)$ is

$\delta V \cdot M_{\mathrm{P}}^{-4} \sim a \delta \operatorname{Vol}(\Sigma) \cdot\left|V_{0} \cdot M_{\mathrm{P}}^{-4}\right| \sim a \delta \operatorname{Vol}(\Sigma) \cdot \frac{\operatorname{Vol}(\Sigma)}{\mathcal{V}^{2}}|\langle\lambda \lambda\rangle|^{2}$,

while

$$
V_{g^{(0,3)}} \cdot M_{\mathrm{P}}^{-4} \sim a \delta \operatorname{Vol}(\Sigma) \cdot \frac{\operatorname{Vol}(\Sigma)^{2}}{\mathcal{V}^{3}}|\langle\lambda \lambda\rangle|^{2} .
$$

Hence the positive contribution coming from $g^{(0,3)}$ is suppressed by the transverse volume and must be neglected. The shift in the volume modulus is related to the warped tension via

$$
a \delta \operatorname{Vol}(\Sigma) \sim \frac{e^{4 \mathcal{A}_{0}} T_{3}}{\operatorname{Vol}(\Sigma)|\langle\lambda \lambda\rangle|^{2}},
$$

which determines the value of $g^{(0,3)}$ to be

$$
g^{(0,3)} \sim e^{-\phi / 2} \frac{e^{4 \mathcal{A}_{0}} T_{3}}{\mathcal{V}}
$$

Therefore, similarly to the situation of nonsupersymmetric classical ISD solutions the value $g^{(0,3)}$ parametrizes SUSY breaking for instance by inducing gaugino mass terms on D7- and D3-branes [81-83].

\section{APPENDIX E: RADIAL PROFILES OF THROAT PERTURBATIONS}

Our analysis relies on the fact that perturbations that are sourced at the bottom of a warped throat fall off towards the bulk Calabi-Yau fast enough to have no significant effect on the integrand of the on-shell potential (47). Therefore, in this appendix we provide details about the radial scaling of throat modes. For our purpose it is sufficient to determine the radial scaling of IR perturbations.

\section{Throat perturbations sourced in the IR}

In order to be able to determine how (47) is affected by a SUSY-breaking source at the bottom of a warped throat, we need to know the approximate radial dependence of field profiles $\phi(r)$ that correspond to perturbations of the KS throat that are sourced in the IR. We do not need to know their field profiles in full detail but only need to estimate the ratio $\phi\left(r_{\mathrm{UV}}\right) / \phi\left(r_{\mathrm{IR}}\right)$ in terms of powers of the warp factor in the IR $\left.e^{\mathcal{A}_{0}} \equiv e^{\mathcal{A}}\right|_{\mathrm{IR}}$ and the $\rho$-modulus mass $m_{\rho}$. This makes the analysis much easier as for this purpose one may approximate parts of the throat by $\mathrm{AdS}_{5} \times T^{1,1}$. In this approximation the field profiles can be determined analytically.

In more detail: away from the tip of the throat the geometry is well approximated by the Klebanov-Tseytlin (KT) solution [101]

$$
d s^{2}=e^{2 \mathcal{A}(r)} d x^{2}+e^{-2 \mathcal{A}}\left(d r^{2}+r^{2} d s_{T^{1,1}}^{2}\right)
$$

with

$$
e^{-4 \mathcal{A}}=L^{4} \frac{\ln \left(r / r_{s}\right)}{r^{4}} .
$$

The KT solution is valid for $r \gg r_{s}$. For our purposes the logarithmic running will be irrelevant and we approximate the throat by $\mathrm{AdS}_{5} \times T^{1,1}$, i.e.

$$
e^{-4 \mathcal{A}} \rightarrow L^{4} / r^{4} .
$$

For this case the analysis of radial profiles of throat perturbation was done for instance in [79].

\section{Scalar perturbations}

Since we are interested in perturbations that are sourced only deep in the IR, only the homogeneous modes of perturbations need to be studied. For scalar perturbations these are solutions to Laplace's equation

$$
\nabla^{2} \phi=0
$$


This is solved by a product ansatz $\phi(r, \Psi)=$ $\sum_{i} \phi_{i}(r) Y_{i}(\Psi)$, where the functions $Y_{i}(\Psi)$ are eigenfunctions of the Laplacian of $T^{1,1}$

$$
\nabla_{T^{1,1}}^{2} Y_{i}=-\lambda_{i}^{2} Y_{i}
$$

Because the 10D Laplacian reads $\nabla^{2}=e^{2 \mathcal{A}}\left[\partial_{r}^{2}+\frac{5}{r} \partial_{r}+\right.$ $\left.\frac{1}{r^{2}} \nabla_{T^{1,1}}^{2}\right]$, the remaining radial equation of motion reads

$$
\partial_{r}^{2} \phi_{i}+\frac{5}{r} \partial_{r} \phi_{i}-\frac{\lambda_{i}^{2}}{r^{2}} \phi_{i}=0,
$$

and is solved by

$$
\phi_{i}(r)=\sum_{i} a_{i} r^{\Delta_{i}-4}+b_{i} r^{-\Delta_{i}}, \quad \Delta_{i} \equiv 2+\sqrt{4+\lambda_{i}^{2}} .
$$

For an infinite throat perturbations that are sourced in the IR are the normalizable ones. Because $\Delta_{i} \geq 4$, the normalizable mode corresponds to $\phi \propto r^{-\Delta_{i}}$. The KK zero mode of $T^{1,1}$ (i.e. the mode with $\lambda_{0}=0$ ) falls off least rapidly towards the UV, i.e.

$$
\phi_{0}\left(r_{\mathrm{UV}}\right) / \phi_{0}\left(r_{\mathrm{IR}}\right) \sim\left(\frac{r_{\mathrm{UV}}}{r_{\mathrm{IR}}}\right)^{-4}=e^{4 \mathcal{A}_{0}}
$$

In the following we would like to see how this behavior is changed when the throat is finite. In this case the would-be non-normalizable mode is not frozen and can in principle play a substantial role as well. This is particularly clear for the KK-zero mode: if it is sourced in the IR it will simply adjust its VEV along the whole throat. In this case its radial profile is determined entirely by the non-normalizable mode.

To discuss this we have to know the UV boundary conditions. Although we do not know these precisely we can encode them in a "UV potential" in the effective 5D theory. First we would like to discuss the KK modes with $\lambda_{i} \neq 0$. For now we set the UV potential to zero, i.e. impose $\left.\partial_{r} \phi_{i}\right|_{r=r_{\mathrm{UV}}}=0$. In this case the solution to the equations of motion can be expressed in terms of the value $\phi_{\mathrm{IR}} \equiv \phi\left(r_{\mathrm{IR}}\right)$ as follows:

$$
\phi(r)=\frac{(\Delta-4) e^{(4-2 \Delta) \mathcal{A}_{0}} r^{-\Delta}+\Delta r^{\Delta-4}}{(\Delta-4) e^{(4-2 \Delta) \mathcal{A}_{0}}+\Delta} \phi_{\mathrm{IR}}
$$

which implies

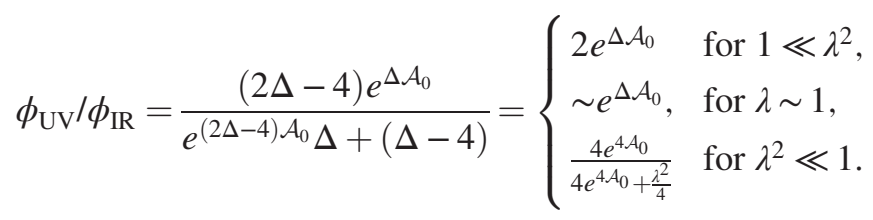

Thus, up to factors of order unity the dependence on the warp factor $e^{\mathcal{A}_{0}}$ can be correctly determined by retaining the normalizable mode only as long as $\lambda^{2} \gg e^{4 \mathcal{A}_{0}}$. For all but the zero mode this is the case. Clearly, including a UV potential can only further reduce $\phi_{\mathrm{UV}} / \phi_{\mathrm{IR}}$.

Next we consider those fields that have no bulk potential (i.e. KK-zero modes of $T^{1,1}$ ) but are stabilized by global effects of the bulk CY, like the axiodilaton in generic flux compactifications. For these fields the UV potential determines the field profile entirely so we cannot neglect it. We approximate the UV potential around its minimum by

$$
V_{\mathrm{UV}}(\phi)=\frac{m_{\mathrm{UV}}^{2}}{2 k} \phi^{2}
$$

Now the UV-boundary condition reads $\left.r \partial_{r} \phi\right|_{r=r_{\mathrm{UV}}}=$ $-\frac{m_{\mathrm{UV}}^{2}}{k^{2}} \phi\left(r_{\mathrm{UV}}\right)$ and the boundary values $\phi_{\mathrm{UV}}$ and $\phi_{\mathrm{IR}}$ are related by

$\phi_{\mathrm{UV}} / \phi_{\mathrm{IR}}=\frac{4 k^{2} e^{4 \mathcal{A}_{0}}}{m_{\mathrm{UV}}^{2}\left(1-e^{4 \mathcal{A}_{0}}\right)}\left(1+\frac{4 k^{2} e^{4 \mathcal{A}_{0}}}{m_{\mathrm{UV}}^{2}\left(1-e^{4 \mathcal{A}_{0}}\right)}\right)^{-1}$

Again, for stabilization $m_{\mathrm{UV}}^{2} / k^{2} \gg e^{4 \mathcal{A}_{0}}$ one obtains

$$
\phi_{\mathrm{UV}} / \phi_{\mathrm{IR}} \approx \frac{4 k^{2} e^{4 \mathcal{A}_{0}}}{m_{\mathrm{UV}}^{2}} .
$$

For the UV potential we may not assume that $m_{\mathrm{UV}} \sim k$. Thus, by only keeping the normalizable mode one would miss a factor of $k^{2} / m_{\mathrm{UV}}^{2}$. In the setting of type IIB flux compactification with only a single Kähler modulus, all fields but the universal Kähler modulus are stabilized perturbatively. This means that all massless bulk fields have UV masses $m_{\mathrm{UV}}^{2} / k^{2}$ that are at most suppressed by the CY volume which we assume to be only moderately large. Therefore, we may estimate the UV tails of perturbations by keeping the normalizable mode only for all throat perturbations.

The universal Kähler modulus $\rho$ plays a very different role: the volume modulus corresponds to a rescaling of the bulk CY where warping is weak. Regions of strong warping are not affected [66]. This means that in the RS1 language the universal Kähler modulus is a UV-brane field stabilized with nonperturbative mass scale $m_{\rho}^{2} \ll k^{2}$ [102]. It couples universally to all UV-perturbations $\delta \phi_{\mathrm{UV}}$,

$$
\mathcal{L}_{\mathrm{UV}} \supset \frac{1}{2} m_{\rho}^{2} \rho^{2}+\Lambda^{3 / 2} \delta \phi_{\mathrm{UV}} \rho+\cdots,
$$

where the coupling $\Lambda^{3 / 2} \delta \phi_{\mathrm{UV}} \rho$ is perturbative and we have omitted higher-order couplings. Then, a shift $\delta \phi_{\text {UV }}$ leads to a shift in $\delta \rho$ of order 


$$
\delta \rho \sim \frac{\Lambda^{3 / 2}}{m_{\rho}^{2}} \delta \phi_{\mathrm{UV}}
$$

Let us work in the regime of moderately large CY volume, strong warping $e^{\mathcal{A}_{0}} \ll 1$, and weak nonperturbative stabilization $m_{\rho}^{2} / k^{2} \ll 1$. Let us further neglect dependencies on the $\mathrm{CY}$ volume and only retain the dependence on $e^{\mathcal{A}_{0}}$ and $m_{\rho}$ which we take to be parametrically small. Then,

$$
\delta \rho \sim \frac{e^{4 \mathcal{A}_{0}}}{m_{\rho}^{2}} \delta \phi_{\mathrm{IR}}
$$

For the nonperturbative volume stabilization of KKLT and uplift with an $\overline{D 3}$-brane of tension $T_{3}$ this implies the (parametric) estimate

$$
\delta \rho \sim \frac{e^{4 \mathcal{A}_{0}} T_{3}}{|\langle\lambda \lambda\rangle|^{2}}
$$

\section{Three-form perturbations}

For the three-form field strength $G_{3}$ the analogous analysis was done in [70]. Setting the dilaton constant for simplicity, the linearized three-form equation of motion and Bianchi identity are

$$
d\left(e^{4 \mathcal{A}} G^{-}\right)=0=d G_{3} .
$$

They are solved by a product ansatz

$$
G_{3}=\sum_{i} d\left(\left(a_{i} r^{\delta_{i}-4}+b_{i} r^{-\delta_{i}}\right) \Omega_{2}^{(i)}\right)
$$

where the $\Omega_{2}^{(i)}$ are eigen-forms of the Laplace-Beltrami operator $*_{5} d$ of $T^{1,1}$,

$$
{ }_{5} d \Omega_{2}^{(i)}=i \delta_{i} \Omega_{2}^{(i)},
$$

with imaginary eigenvalues $i \delta_{i}$.

In [70] it is found that the ISD/IASD pieces are given by

$G^{+}=G_{0}^{+}+\frac{i}{2} \sum_{i \neq 0}\left(\frac{a_{i}}{\delta_{i}} r^{\delta_{i}-4}+2 b_{i} r^{-\delta_{i}}\right)\left(d \Omega_{2}^{(i)}-\delta_{i} \frac{d r}{r} \wedge \Omega_{2}^{(i)}\right)$,

$G^{-}=G_{0}^{-}-i \sum_{i \neq 0} a_{i} \frac{\delta_{i}-2}{\delta_{i}} r^{\delta_{i}-4}\left(d \Omega_{2}^{(i)}+\delta_{i} \frac{d r}{r} \wedge \Omega_{2}^{(i)}\right)$,

where the zero mode pieces are given by

$$
G_{0}^{ \pm}=-2 a_{0} r^{-4}\left(*_{5} \Omega_{2}^{(0)} \pm i \frac{d r}{r} \wedge \Omega_{2}^{(0)}\right)
$$

Only those eigen two-forms with $\delta_{i}<2$ give rise to normalizable IASD perturbations. The lowest eigenvalues of the Laplace-Beltrami operator are listed in tables 3-5 of [70]. It can be read off that the zero mode falls off least rapidly towards the UV, namely as $r^{-4}$. For ISD perturbations the story is a bit different as also those modes that correspond to the coefficients $b_{i}$ contribute. Those that feature $\delta_{i}>2$ are normalizable. The one that falls off least rapidly towards the UV corresponds to $\delta=3$ and has a radial profile that is proportional to $r^{-3}$ [70]. This corresponds to the two-form

$$
\Omega_{2}^{(\delta=3)}=\left(g^{1}+i g^{4}\right) \wedge\left(g^{2}-i g^{3}\right),
$$

using the basis of one-forms of $T^{1,1}$ used in [51]. Far from the tip, the KS solution can be viewed as a perturbation of the KT solution and the normalizable $\delta=3$ mode precisely encodes the leading perturbation that is sourced by the deformation at the tip [103]. Hence, it is of Hodge type $(2,1)$ and does not enter (47). As a consequence, the perturbation of three-form fluxes on (47) is irrelevant to the order of precision we are interested in, analogous to the scalar case.

\section{Metric perturbations}

In [79] the radial scaling of metric modes was derived. After gauge fixing, the only nonvanishing modes are those that correspond to transverse traceless two-tensors of $T^{1,1}$,

$$
\begin{aligned}
\delta g_{i j} & \sim \sum_{n}\left(a_{n} r^{\Delta_{n}(g)-4}+b_{n} r^{-\Delta_{n}(g)}\right) Y_{i j}^{(n)}, \\
\Delta_{n}(g) & \equiv 2+\sqrt{\hat{\lambda}_{n}^{2}-4},
\end{aligned}
$$

where the $Y_{i j}^{(n)}$ are the transverse traceless eigentensors of the Lichnerowicz operator of $T^{1,1}$ with eigenvalue $\hat{\lambda}_{n}^{2}$,

$$
\nabla^{2} Y_{i j}^{(i)}-2 \nabla^{k} \nabla_{(i} Y_{j) k}^{(i)}=-\hat{\lambda}_{n}^{2} Y_{i j}^{(i)}
$$

In [80] it is found that the lowest eigenvalues are $\hat{\lambda}^{2}=$ $4,5,36-8 \sqrt{7}, 65 / 4,20, \ldots$ corresponding to

$$
\Delta_{n}(g)=2,3,5.29,11 / 2,6 \ldots
$$

The dominant normalizable mode thus falls off as $r^{-3}$ and corresponds to the deformation of the conifold. All further perturbations fall off at least as $r^{-5.29}$. 
[1] S. Weinberg, The cosmological constant problem, Rev. Mod. Phys. 61, 1 (1989).

[2] N. Kaloper and A. Padilla, Sequestering the Standard Model Vacuum Energy, Phys. Rev. Lett. 112, 091304 (2014).

[3] N. Kaloper, A. Padilla, D. Stefanyszyn, and G. Zahariade, Manifestly Local Theory of Vacuum Energy Sequestering, Phys. Rev. Lett. 116, 051302 (2016).

[4] N. Kaloper and A. Padilla, Vacuum Energy Sequestering and Graviton Loops, Phys. Rev. Lett. 118, 061303 (2017).

[5] A. D. Linde, The inflationary universe, Rep. Prog. Phys. 47, 925 (1984).

[6] A. D. Linde, Inflation and Quantum Cosmology (unpublished).

[7] A. D. Linde, in Three Hundred Years of Gravitation, edited by S. W. Hawking and W. Israel (Cambridge University Press, 1987), p. 604.

[8] S. Weinberg, Anthropic Bound on the Cosmological Constant, Phys. Rev. Lett. 59, 2607 (1987).

[9] J. D. Barrow and F. J. Tipler, The Anthropic Cosmological Principle ( Oxford University Press, Oxford, England, 1988).

[10] A. Vilenkin, Birth of inflationary universes, Phys. Rev. D 27, 2848 (1983).

[11] A. D. Linde, Eternally existing self-reproducing chaotic inflationary universe, Phys. Lett. B 175, 395 (1986).

[12] L. Susskind, The anthropic landscape of string theory, arXiv:hep-th/0302219.

[13] S. Kachru, R. Kallosh, A. D. Linde, and S. P. Trivedi, De Sitter vacua in string theory, Phys. Rev. D 68, 046005 (2003).

[14] S. B. Giddings, S. Kachru, and J. Polchinski, Hierarchies from fluxes in string compactifications, Phys. Rev. D 66, 106006 (2002).

[15] K. Dasgupta, G. Rajesh, and S. Sethi, M theory, orientifolds and G- flux, J. High Energy Phys. 08 (1999) 023.

[16] S. Kachru, J. Pearson, and H. L. Verlinde, Brane/flux annihilation and the string dual of a nonsupersymmetric field theory, J. High Energy Phys. 06 (2002) 021.

[17] G. Veneziano and S. Yankielowicz, An effective Lagrangian for the pure $N=1$ supersymmetric Yang-Mills theory, Phys. Lett. 113B, 231 (1982).

[18] S. Ferrara, L. Girardello, and H. P. Nilles, Breakdown of local supersymmetry through gauge fermion condensates, Phys. Lett. 125B, 457 (1983).

[19] I. Affleck, M. Dine, and N. Seiberg, Dynamical supersymmetry breaking in supersymmetric QCD, Nucl. Phys. B241, 493 (1984).

[20] M. Dine, R. Rohm, N. Seiberg, and E. Witten, Gluino Condensation in superstring models, Phys. Lett. 156B, 55 (1985).

[21] B. Freivogel and M. Lippert, Evidence for a bound on the lifetime of de Sitter space, J. High Energy Phys. 12 (2008) 096.

[22] J. Blaback, U. H. Danielsson, and T. Van Riet, Resolving anti-brane singularities through time-dependence, J. High Energy Phys. 02 (2013) 061.

[23] I. Bena, D. Junghans, S. Kuperstein, T. Van Riet, T. Wrase, and M. Zagermann, Persistent anti-brane singularities, J. High Energy Phys. 10 (2012) 078.
[24] I. Bena, M. Grana, S. Kuperstein, and S. Massai, Polchinski-Strassler does not uplift Klebanov-Strassler, J. High Energy Phys. 09 (2013) 142.

[25] I. Bena, A. Buchel, and O. J. C. Dias, Horizons cannot save the Landscape, Phys. Rev. D 87, 063012 (2013).

[26] I. Bena, J. Blaback, U. H. Danielsson, and T. Van Riet, Antibranes cannot become black, Phys. Rev. D 87, 104023 (2013).

[27] D. Junghans, D. Schmidt, and M. Zagermann, Curvatureinduced resolution of anti-brane singularities, J. High Energy Phys. 10 (2014) 34.

[28] I. Bena, M. Grana, S. Kuperstein, and S. Massai, Giant tachyons in the landscape, J. High Energy Phys. 02 (2015) 146.

[29] U. H. Danielsson and T. Van Riet, Fatal attraction: More on decaying anti-branes, J. High Energy Phys. 03 (2015) 087.

[30] I. Bena and S. Kuperstein, Brane polarization is no cure for tachyons, J. High Energy Phys. 09 (2015) 112.

[31] D. Cohen-Maldonado, J. Diaz, T. van Riet, and B. Vercnocke, Observations on fluxes near anti-branes, J. High Energy Phys. 01 (2016) 126.

[32] G. S. Hartnett, Localised anti-branes in flux backgrounds, J. High Energy Phys. 06 (2015) 007.

[33] U.H. Danielsson, Perturbative decay of anti-branes in flux backgrounds due to space time instabilities, arXiv: 1502.01234.

[34] B. Michel, E. Mintun, J. Polchinski, A. Puhm, and P. Saad, Remarks on brane and antibrane dynamics, J. High Energy Phys. 09 (2015) 021.

[35] J. Polchinski, Brane/antibrane dynamics and KKLT stability, arXiv:1509.05710.

[36] X. Dong, B. Horn, E. Silverstein, and A. Westphal, Simple exercises to flatten your potential, Phys. Rev. D 84, 026011 (2011).

[37] R. Kallosh and A. D. Linde, Landscape, the scale of SUSY breaking, and inflation, J. High Energy Phys. 12 (2004) 004.

[38] V. Balasubramanian, P. Berglund, J. P. Conlon, and F. Quevedo, Systematics of moduli stabilisation in CalabiYau flux compactifications, J. High Energy Phys. 03 (2005) 007.

[39] C. P. Burgess, R. Kallosh, and F. Quevedo, De Sitter string vacua from supersymmetric D terms, J. High Energy Phys. 10 (2003) 056.

[40] A. Saltman and E. Silverstein, A new handle on de Sitter compactifications, J. High Energy Phys. 01 (2006) 139.

[41] A. Westphal, de Sitter string vacua from Kahler uplifting, J. High Energy Phys. 03 (2007) 102.

[42] M. Cicoli, A. Maharana, F. Quevedo, and C. P. Burgess, De Sitter string vacua from dilaton-dependent non-perturbative effects, J. High Energy Phys. 06 (2012) 011.

[43] J. Louis, M. Rummel, R. Valandro, and A. Westphal, Building an explicit de Sitter, J. High Energy Phys. 10 (2012) 163.

[44] M. Rummel and Y. Sumitomo, De Sitter vacua from a D-term generated racetrack uplift, J. High Energy Phys. 01 (2015) 015.

[45] M. Cicoli, F. Quevedo, and R. Valandro, De Sitter from T-branes, J. High Energy Phys. 03 (2016) 141. 
[46] A. P. Braun, M. Rummel, Y. Sumitomo, and R. Valandro, De Sitter vacua from a D-term generated racetrack potential in hypersurface Calabi-Yau compactifications, J. High Energy Phys. 12 (2015) 033.

[47] A. Retolaza and A. Uranga, De Sitter uplift with dynamical SUSY breaking, J. High Energy Phys. 04 (2016) 137.

[48] D. Gallego, M. C. D. Marsh, B. Vercnocke, and T. Wrase, A new class of de Sitter vacua in type IIB large volume compactifications, J. High Energy Phys. 10 (2017) 193.

[49] S. Gukov, C. Vafa, and E. Witten, CFT's from Calabi-Yau four folds, Nucl. Phys. B584, 69 (2000); Erratum, Nucl. Phys. B608, 477 (2001).

[50] E. Witten, Nonperturbative superpotentials in string theory, Nucl. Phys. B474, 343 (1996).

[51] I. R. Klebanov and M. J. Strassler, Supergravity and a confining gauge theory: Duality cascades and chi SB resolution of naked singularities, J. High Energy Phys. 08 (2000) 052.

[52] Z. Komargodski and N. Seiberg, From linear SUSY to constrained superfields, J. High Energy Phys. 09 (2009) 066.

[53] D. V. Volkov and V. P. Akulov, Is the neutrino a Goldstone particle?, Phys. Lett. 46B, 109 (1973).

[54] K. Choi, A. Falkowski, H. P. Nilles, and M. Olechowski, Soft supersymmetry breaking in KKLT flux compactification, Nucl. Phys. B718, 113 (2005).

[55] O. Lebedev, H. P. Nilles, and M. Ratz, De Sitter vacua from matter superpotentials, Phys. Lett. B 636, 126 (2006).

[56] F. Brummer, A. Hebecker, and M. Trapletti, SUSY breaking mediation by throat fields, Nucl. Phys. B755, 186 (2006).

[57] I. Antoniadis, E. Dudas, S. Ferrara, and A. Sagnotti, The Volkov-Akulov-Starobinsky supergravity, Phys. Lett. B 733, 32 (2014).

[58] S. Ferrara, R. Kallosh, and A. Linde, Cosmology with nilpotent superfields, J. High Energy Phys. 10 (2014) 143.

[59] R. Kallosh and T. Wrase, Emergence of spontaneously broken supersymmetry on an anti-D3-brane in KKLT dS vacua, J. High Energy Phys. 12 (2014) 117.

[60] E. A. Bergshoeff, K. Dasgupta, R. Kallosh, A. Van Proeyen, and T. Wrase, $\overline{\mathrm{D} 3}$ and dS, J. High Energy Phys. 05 (2015) 058.

[61] R. Kallosh, F. Quevedo, and A. M. Uranga, String theory realizations of the nilpotent Goldstino, J. High Energy Phys. 12 (2015) 039.

[62] R. Kallosh, A. Linde, D. Roest, and Y. Yamada, $\overline{D 3}$ induced geometric inflation, J. High Energy Phys. 07 (2017) 057.

[63] M. P. Garcia del Moral, S. Parameswaran, N. Quiroz, and I. Zavala, Anti-D3 branes and moduli in non-linear supergravity, J. High Energy Phys. 10 (2017) 185.

[64] M. Berg, M. Haack, and B. Kors, Loop corrections to volume moduli and inflation in string theory, Phys. Rev. D 71, 026005 (2005).

[65] D. Baumann, A. Dymarsky, I. R. Klebanov, J. M. Maldacena, L.P. McAllister, and A. Murugan, On D3-brane potentials in compactifications with fluxes and wrapped D-branes, J. High Energy Phys. 11 (2006) 031.
[66] S. B. Giddings and A. Maharana, Dynamics of warped compactifications and the shape of the warped landscape, Phys. Rev. D 73, 126003 (2006).

[67] J. M. Maldacena and C. Nunez, Supergravity description of field theories on curved manifolds and a no go theorem, Int. J. Mod. Phys. A 16, 822 (2001).

[68] P. G. O. Freund and M. A. Rubin, Dynamics of dimensional reduction, Phys. Lett. 97B, 233 (1980).

[69] M. Grana, R. Minasian, M. Petrini, and A. Tomasiello, Supersymmetric backgrounds from generalized CalabiYau manifolds, J. High Energy Phys. 08 (2004) 046.

[70] D. Baumann, A. Dymarsky, S. Kachru, I. R. Klebanov, and L. McAllister, D3-brane potentials from fluxes in AdS/CFT, J. High Energy Phys. 06 (2010) 072.

[71] D. Baumann, A. Dymarsky, I. R. Klebanov, and L. McAllister, Towards an explicit model of D-brane inflation, J. Cosmol. Astropart. Phys. 01 (2008) 024.

[72] A. Dymarsky and L. Martucci, D-brane non-perturbative effects and geometric deformations, J. High Energy Phys. 04 (2011) 061.

[73] B. Heidenreich, L. McAllister, and G. Torroba, Dynamic SU(2) structure from seven-branes, J. High Energy Phys. 05 (2011) 110.

[74] A. R. Frey and M. Lippert, AdS strings with torsion: Noncomplex heterotic compactifications, Phys. Rev. D 72, 126001 (2005).

[75] P. Koerber and L. Martucci, From ten to four and back again: How to generalize the geometry, J. High Energy Phys. 08 (2007) 059.

[76] P. Koerber and L. Martucci, Warped generalized geometry compactifications, effective theories and non-perturbative effects, Fortschr. Phys. 56, 862 (2008).

[77] L. Randall and R. Sundrum, A Large mass hierarchy from a small extra dimension, Phys. Rev. Lett. 83, 3370 (1999).

[78] A. Sen, F theory and orientifolds, Nucl. Phys. B475, 562 (1996).

[79] S. Gandhi, L. McAllister, and S. Sjors, A toolkit for perturbing flux compactifications, J. High Energy Phys. 12 (2011) 053.

[80] S. Gandhi, L. McAllister, and S. Sjors, On antibrane moduli in stabilized compactifications (unpublished).

[81] P. G. Camara, L. E. Ibanez, and A. M. Uranga, Flux induced SUSY breaking soft terms, Nucl. Phys. B689, 195 (2004).

[82] M. Grana, T. W. Grimm, H. Jockers, and J. Louis, Soft supersymmetry breaking in Calabi-Yau orientifolds with D-branes and fluxes, Nucl. Phys. B690, 21 (2004).

[83] P. G. Camara, L. E. Ibanez, and A. M. Uranga, Fluxinduced SUSY-breaking soft terms on D7-D3 brane systems, Nucl. Phys. B708, 268 (2005).

[84] K. Becker, M. Becker, M. Haack, and J. Louis, Supersymmetry breaking and alpha-prime corrections to flux induced potentials, J. High Energy Phys. 06 (2002) 060.

[85] F. Bonetti and M. Weissenbacher, The Euler characteristic correction to the Kähler potential-revisited, J. High Energy Phys. 01 (2017) 003.

[86] R. Minasian, T. G. Pugh, and R. Savelli, F-theory at order $\alpha^{\prime 3}$, J. High Energy Phys. 10 (2015) 050.

[87] J. P. Conlon, F. Quevedo, and K. Suruliz, Large-volume flux compactifications: Moduli spectrum and D3/D7 soft 
supersymmetry breaking, J. High Energy Phys. 08 (2005) 007.

[88] G. von Gersdorff and A. Hebecker, Kahler corrections for the volume modulus of flux compactifications, Phys. Lett. B 624, 270 (2005).

[89] M. Berg, M. Haack, and B. Kors, String loop corrections to Kahler potentials in orientifolds, J. High Energy Phys. 11 (2005) 030.

[90] M. Berg, M. Haack, and E. Pajer, Jumping through loops: On soft terms from large volume compactifications, J. High Energy Phys. 09 (2007) 031.

[91] M. Cicoli, J. P. Conlon, and F. Quevedo, Systematics of string loop corrections in type IIB Calabi-Yau flux compactifications, J. High Energy Phys. 01 (2008) 052.

[92] M. Cicoli, J. P. Conlon, and F. Quevedo, General analysis of LARGE volume scenarios with string loop moduli stabilisation, J. High Energy Phys. 10 (2008) 105.

[93] M. Berg, M. Haack, J. U. Kang, and S. Sjörs, Towards the one-loop Kähler metric of Calabi-Yau orientifolds, J. High Energy Phys. 12 (2014) 077.

[94] D. Lust, F. Marchesano, L. Martucci, and D. Tsimpis, Generalized non-supersymmetric flux vacua, J. High Energy Phys. 11 (2008) 021.
[95] D. Marolf, L. Martucci, and P. J. Silva, Fermions, T duality and effective actions for D-branes in bosonic backgrounds, J. High Energy Phys. 04 (2003) 051.

[96] D. Marolf, L. Martucci, and P. J. Silva, Actions and fermionic symmetries for D-branes in bosonic backgrounds, J. High Energy Phys. 07 (2003) 019.

[97] L. Martucci, J. Rosseel, D. Van den Bleeken, and A. Van Proeyen, Dirac actions for D-branes on backgrounds with fluxes, Classical Quantum Gravity 22, 2745 (2005).

[98] H. Federer, Geometric Measure Theory (Springer, Berlin, 1969).

[99] R. Harvey and H. B. Lawson, Jr., Calibrated geometries, Acta Math. 148, 47 (1982).

[100] P. Koerber, Stable D-branes, calibrations and generalized Calabi-Yau geometry, J. High Energy Phys. 08 (2005) 099.

[101] I. R. Klebanov and A. A. Tseytlin, Gravity duals of supersymmetric $\mathrm{SU}(\mathrm{N}) \times \mathrm{SU}(\mathrm{N}+\mathrm{M})$ gauge theories, Nucl. Phys. B578, 123 (2000).

[102] F. Brummer, A. Hebecker, and E. Trincherini, The throat as a Randall-Sundrum model with Goldberger-Wise stabilization, Nucl. Phys. B738, 283 (2006).

[103] A. Loewy and J. Sonnenschein, On the holographic duals of $N=1$ gauge dynamics, J. High Energy Phys. 08 (2001) 007. 